\title{
ASYMPTOTIC APPROXIMATIONS FOR THE JACOBI AND ULTRASPHERICAL POLYNOMIALS, AND RELATED FUNCTIONS*
}

\author{
T. M. DUNSTER ${ }^{\dagger}$
}

\author{
To Richard Askey on his 65th birthday
}

\begin{abstract}
Uniform asymptotic approximations are derived for three fundamental solutions of Jacobi's differential equation, one of which is the Jacobi polynomial $P_{n}^{(p, q)}(x)$ when the parameter $n$ is an integer. The case where the parameters $p, q$, and $n$ appearing in the equation are real and nonnegative is considered (with $n$ not necessarily an integer), and where the argument $x$ can lie in unbounded complex domains. The results are obtained by an application of existing asymptotic theories of a coalescing turning point and simple pole in the complex plane, and of a coalescing turning point and double pole in the complex plane. The resulting approximations achieve a uniform reduction of free variables from three to two, and involve either Whittaker's confluent hypergeometric functions or Bessel functions. Four cases are considered: (i) $q$ large, $p$ fixed, and $n$ taking any value in the range $0 \leq n \leq O(q)$, (ii) $n$ large, $p$ fixed, and $q$ taking any value in the range $0 \leq q \leq O(n)$, (iii) $p$ and $q$ large and equal, with $n$ taking any value in the range $0 \leq n \leq O(p)$, and (iv) $n$ large, with $p$ and $q$ equal and taking any value in the range $0 \leq p=q \leq O(n)$. In cases (i) and (ii), the roles of $p$ and $q$ can be interchanged via well-known connection formulas (which are provided), and cases (iii) and (iv) provide asymptotic approximations for the ultraspherical (Gegenbauer) polynomials when $n$ is an integer. Explicit error bounds are available for all the approximations.
\end{abstract}

1. Introduction. In this paper, we shall examine the asymptotic behavior of solutions of Jacobi's differential equation

$$
\left(1-x^{2}\right) \frac{d^{2} y}{d x^{2}}+[(q-p)-(p+q+2) x] \frac{d y}{d x}+n(n+p+q+1) y=0
$$

which is characterized by having regular singularities at $x= \pm 1$ and $x=\infty$. We seek asymptotic approximations for nonnegative values of the parameters for the case where

$$
u \equiv n+\frac{1}{2}(p+q+1) \rightarrow \infty
$$

with one or more of the parameters $n, p$, and $q$ being considered large.

We shall consider the following four cases. In $\S 2$, we obtain asymptotic approximations for $q$ large, $p$ fixed, and $n$ taking any value in the range $0 \leq n \leq O(q)$. In $\S 3$, we obtain asymptotic expansions for the case $n$ large, $p$ fixed, and $q$ taking any value in the range $0 \leq q \leq O(n)$. In $\S \S 4$ and 5 , we consider the case where $p$ and $q$ are equal: in $\S 4$, we assume $p$ and $q$ are large, with $n$ taking any value in the range $0 \leq n \leq O(p)$, and in $\S 5 n$ is considered large with $p$ and $q$ taking any value in the range $0 \leq p=q \leq O(n)$. With the aid of the connection formulas (1.23) and (1.24) given below if necessary, the asymptotic approximations of $\S \S 2,4$, and 5 are valid for all complex $x$, and the asymptotic expansions of $\S 3$ are valid for all $x$ except at points near the singularity at $x=1$. The roles of $p$ and $q$ can be interchanged in the results of $\S \S 2$ and 3 again by an appropriate use of the connection formulas (1.23) and (1.24).

${ }^{*}$ Received June 26, 1998, revised 18 February, 1999.

$\dagger$ Department of Mathematical Sciences, San Diego State University, San Diego, CA 92182-7720, USA (dunster@math.sdsu.edu). 
When $n \in \mathbf{N}$, the most important solution of (1.1) is the Jacobi polynomial, given by

$$
P_{n}^{(p, q)}(x)=\frac{\Gamma(n+p+1)}{n ! \Gamma(n+p+q+1)} \sum_{k=0}^{n}\left(\begin{array}{l}
n \\
k
\end{array}\right) \frac{\Gamma(n+p+q+k+1)}{\Gamma(p+k+1)}\left(\frac{x-1}{2}\right)^{k} .
$$

Jacobi polynomials are one of the class of classical orthogonal polynomials (which also include Legendre, Laguerre, Hermite, Chebyshev, and Gegenbauer polynomials), and satisfy the orthogonality condition

$$
\int_{-1}^{1}\left[(1-x)^{p}(1+x)^{q}\right] P_{n}^{(p, q)}(x) P_{m}^{(p, q)}(x) d x=0, \quad m \neq n .
$$

Amongst their many important applications, we mention their role in the interactions of special functions and group theory[2]. In addition to $P_{n}^{(p, q)}(x)$, we shall consider, in this paper, other solutions of (1.1), as well as the more general case when $n$ is not necessarily an integer.

There have been many investigations into the asymptotics of solutions of Jacobi's equation (1.1), although most have focused on the Jacobi polynomial (1.2) (see for example [3], [5], [7], [9], and [12]). Frenzen and Wong [9] used an integral representation to get an approximation (with error bounds) for the Jacobi polynomial in terms of Bessel functions, for $u \rightarrow \infty$ with $p, q$ fixed and $-1+\delta \leq x \leq 1$. These approximations were used subsequently by Qu and Wong [13] to settle Szegö's 1926 conjecture on the monotonicity of Lebesgue constants for Legendre series.

In 1989, Chen and Ismail derived approximations for $P_{n}^{(A+B n, C+D n)}(x)$ as $n \rightarrow \infty$ with $A, B, C, D$, and $x$ all remaining fixed. Asymptotic approximations for a second solution of (1.1), which at infinity is recessive (see [10, Chap.5, 7]), have been obtained by Elliot [7] and Nestor [12], and most recently by Wong and Zhang [16]. Also Wimp et al. [15] considered the numerical computation of an associated function which is real-valued on the interval $(-1,1)$. Nestor's [12] approximations are valid for real $x$ in the interval $(1, \infty)$ and for a large range of the parameters, and include error bounds. The results of $\S 2$ of the present paper are an extension of his results to all values of $x$ (real and complex). Most recently, Wong and Zhang [16] extended the results of Elliot [7]; they employed an asymptotic theory of Olver [10, Chap.12], involving differential equations having a simple pole, to derive an asymptotic expansion of a second solution to the Jacobi differential equation, along with a two-term asymptotic approximation for its zeros.

Returning to Jacobi's equation (1.1), we note that for $n \in \mathbf{N}$ and $p=q=$ $\pm \frac{1}{2}$, we obtain, as a special case, the Chebyshev polynomials as solutions. More genera.ly, when $n \in \mathbf{N}$ and $p$ and $q$ are equal, solutions of (1.1) are the ultraspherical (Gegenbauer) polynomials $C_{n}^{(r)}(x)$, which in terms of (1.3) are given by

$$
C_{n}^{(r)}(x)=\frac{\Gamma\left(r+\frac{1}{2}\right) \Gamma(2 r+n)}{\Gamma(2 r) \Gamma\left(r+n+\frac{1}{2}\right)} P_{n}^{\left(r-\frac{1}{2}, r-\frac{1}{2}\right)}(x) .
$$

Asymptotic approximations for these polynomials can be obtained directly from the results of $\S 4$ below.

When $n$ is not an integer, $P_{n}^{(p, q)}(x)$ is no longer a polynomial, and (1.3) generalizes to

$$
P_{n}^{(p, q)}(x)=\frac{\Gamma(n+p+1)}{\Gamma(n+1) \Gamma(p+1)} F\left(-n, n+p+q+1 ; p+1 ; \frac{1}{2}(1-x)\right)
$$


where $F$ as usual denotes the hypergeometric function. Thus, in general, $P_{n}^{(p, q)}(x)$ has a branch point, or pole, at $x=-1$ and $x=\infty$. Its principal branch is restricted to the $x$ plane having a cut along the negative real axis from $x=-1$ and $x=\infty$, with other branches obtainable by continuation across this cut.

As we remarked above, we shall consider real non-negative values of the three parameters $n, p$, and $q$, and in this case, $P_{n}^{(p, q)}(x)$ is uniquely characterized as being recessive at $x=1(\arg (x)=0)$. It is always dominant at infinity and is recessive at $x=-1$ if and only if $n \in \mathbf{N}$.

We consider two companion solutions to $P_{n}^{(p, q)}(x)$. The first, which is recessive at infinity, is defined by Szegö [14]

$$
Q_{n}^{(p, q)}(x)=\frac{1}{2^{n+1}(x-1)^{p}(x+1)^{q}} \int_{-1}^{1} \frac{(1-t)^{n+p}(1+t)^{n+q}}{(x-t)^{n+1}} d t, \quad x \notin[-1,1]
$$

or, as an alternative representation (see [7], [8], and [12]),

$$
Q_{n}^{(p, q)}(x)=\frac{1}{2(x-1)^{p}(x+1)^{q}} \int_{-1}^{1} \frac{(1-t)^{p}(1+t)^{q}}{x-t} P_{n}^{(p, q)}(t) d t, \quad x \notin[-1,1] .
$$

The representation we find most useful is in terms of the hypergeometric function

$$
\begin{aligned}
Q_{n}^{(p, q)}(x)=K_{n}^{(p, q)} & \left(\frac{2}{x-1}\right)^{n+p+q+1} \\
& \times F\left(n+p+q+1, n+q+1 ; 2 n+p+q+2 ; 2(1-x)^{-1}\right)
\end{aligned}
$$

in which for later convenience we have defined,

$$
K_{n}^{(p, q)}=\frac{\Gamma(n+p+1) \Gamma(n+q+1)}{2 \Gamma(2 n+p+q+2)} .
$$

The hypergeometric function in the representation (1.9) has branch points at $x= \pm 1$ and, along with the factor $(x-1)^{-n-p-q-1}$, takes its principal value in the $x$ plane having a cut along $(-\infty, 1]$.

We shall let $y\left(x e^{\pi i}\right)\left(y\left(x e^{-\pi i}\right)\right)$ denote the branch of any solution $y(x)$ of Jacobi's equation obtained from the principal branch by making a positive (negative) halfcircuit of the ellipse having \pm 1 as foci and passing through $x$. Now, since Jacobi's equation (1.1) is unchanged when $x$ is replaced by $-x$, with $p$ and $q$ interchanged, it follows that $P_{n}^{(q, p)}\left(x e^{-\pi i}\right)$ is also a solution of the equation. We shall take this as the third fundamental solution, and since it is recessive at $x=-1(\arg (x)=\pi)$, it comes into play when $n$ is not an integer. Note that $P_{n}^{(q, p)}\left(x e^{-\pi i}\right)$ has its principal branch taken in the $x$ plane having a cut along $[1, \infty)$. We remark that when $n \in \mathbf{N}$, the solutions $P_{n}^{(q, p)}\left(x e^{-\pi i}\right)$ and $P_{n}^{(p, q)}(x)$ are linearly dependent polynomials, and in particular,

$$
P_{n}^{(p, q)}(-x)=(-1)^{n} P_{n}^{(q, p)}(x) .
$$

We now record various results involving these three solutions which will be of use later. First consider the asymptotic behavior of the solutions at the three singularities of Jacobi's equation (1.1). As $x \rightarrow 1(\arg (x)=0)$

$$
P_{n}^{(p, q)}(x)=\frac{\Gamma(n+p+1)}{\Gamma(n+1) \Gamma(p+1)}\{1+O(x-1)\}
$$


and as $x \rightarrow 1^{+}(\arg (x)=0)$

$$
Q_{n}^{(p, q)}(x)=\frac{1}{2}\left\{\frac{\Gamma(p) \Gamma(n+q+1)}{\Gamma(n+p+q+1)}\left(\frac{2}{x-1}\right)^{p}+\frac{\Gamma(n+p+1) \Gamma(-p)}{\Gamma(n+1)}\right\}\{1+O(x-1)\},
$$

in the latter case provided $p \neq 0,1,2, \ldots$. If $p=1,2,3, \ldots$, then from (1.9) and [1, Eq. 15.3.14], we find as $x \rightarrow 1^{+}$

$$
\begin{aligned}
Q_{n}^{(p, q)}(x)=\frac{\Gamma(p) \Gamma(n+q+1)}{2 \Gamma(n+p+q+1)}\left(\frac{2}{x-1}\right)^{p} \\
\quad+\frac{\sin \{(n+p) \pi\} \Gamma(n+p+1)}{2 \sin (n \pi) \Gamma(n+1) \Gamma(p+1)} \ln \left(\frac{2}{x-1}\right)+O\left\{(x-1)^{-p+1}\right\}
\end{aligned}
$$

where the limit is taken on the second term on the right-hand side when $n$ is an integer or zero. If $p=0$, then

$$
Q_{n}^{(0, q)}(x)=\frac{1}{2} \ln \left(\frac{2}{x-1}\right)-\gamma-\frac{1}{2} \psi(n+q+1)-\frac{1}{2} \psi(n+1)+O\{(x-1) \ln (x-1)\}
$$

where $\psi(z)=\Gamma^{\prime}(z) / \Gamma(z)$ is the digamma function, and $\gamma=-\psi(1)$ is Euler's constant. Next, as $x \rightarrow-1^{+}$(in the principal plane)

$$
P_{n}^{(p, q)}(x)=-\frac{\sin (n \pi) \Gamma(n+p+1) \Gamma(q)}{\pi \Gamma(n+p+q+1)}\left(\frac{2}{x+1}\right)^{q}\{1+O(x+1)\},
$$

provided that $n$ is not an integer and $q>0$ and $q \neq 1$; in the latter case, (1.16) still holds, but the $O(x+1)$ term must be replaced by $O\{(x+1) \ln (x+1)\}$. If $q=0$ (and $n$ is not an integer), then

$$
\begin{gathered}
P_{n}^{(p, 0)}(x)=\frac{\sin (n \pi)}{\pi}\left[\ln \left\{\frac{1}{2}(x+1)\right\}+2 \gamma+\psi(n+p+1)+\psi(n+1)\right] \\
+\cos (n \pi)+O\{(x+1) \ln (x+1)\},
\end{gathered}
$$

and finally, if $n$ is an integer, then

$$
P_{n}^{(p, q)}(x)=(-1)^{n} \frac{\Gamma(n+q+1)}{\Gamma(n+1) \Gamma(q+1)}\{1+O(x+1)\} .
$$

As $x \rightarrow-1 \pm i 0(\arg (x)= \pm \pi)$ and $q>0$,

$$
Q_{n}^{(p, q)}(x)=-e^{\mp(n+p) \pi i} \frac{\Gamma(n+p+1) \Gamma(q)}{2 \Gamma(n+p+q+1)}\left(\frac{2}{x+1}\right)^{q}\{1+O(x+1)\},
$$

except when $q=1$, in which case the $O(x+1)$ term must be replaced by $O(x+1)$ $\ln (x+1)$. If $q=0$, then

$$
Q_{n}^{(p, 0)}(x)=\frac{1}{2} e^{\mp(n+p) \pi i}\left[\ln \left\{\frac{1}{2}(x+1)\right\}+2 \gamma+\psi(n+p+1)+\psi(n+1) \mp \pi i\right]
$$

$$
+O\{(x+1) \ln (x+1)\} \text {. }
$$


Next, as $x \rightarrow \infty$,

$$
Q_{n}^{(p, q)}(x)=K_{n}^{(p, q)}\left(\frac{2}{x}\right)^{n+p+q+1}\left\{1+O\left(x^{-1}\right)\right\}
$$

and

$$
P_{n}^{(p, q)}(x) \sim \frac{\Gamma(2 n+p+q+1)}{\Gamma(n+p+q+1) \Gamma(n+1)}\left(\frac{x}{2}\right)^{n},
$$

with principal branches applying in both cases.

We also shall find the following connection formulas very useful:

$$
P_{n}^{(p, q)}(x)=e^{\mp n \pi i} P_{n}^{(q, p)}\left(x e^{ \pm \pi i}\right)+\frac{2 e^{\mp(n+p) \pi i} \sin (n \pi)}{\pi} Q_{n}^{(p, q)}(x)
$$

and

$$
Q_{n}^{(q, p)}\left(x e^{ \pm \pi i}\right)=-e^{\mp(n+p+q) \pi i} Q_{n}^{(p, q)}(x) .
$$

We also note the continuation of the solutions around the singularity $x=-1$. Suppose that $x$ lies on the upper part of a cut from $[-1,1]$ with $\arg (x+1)=0$ and $x \neq \pm 1$; we let $-1+(x+1) e^{2 \pi i}$ denote the corresponding point on the lower part of the cut where $\arg \left\{-1+(x+1) e^{2 \pi i}\right\}=2 \pi$ (i.e., the point obtained by encircling the singularity $x=-1$ once in the positive sense). Then from (1.6) and [10, Chap.5, Eq.(10.12)], we find that

$$
\begin{aligned}
P_{n}^{(p, q)}\left(-1+(x+1) e^{2 \pi i}\right)=e^{-2 q \pi i} P_{n}^{(p, q)} & (x) \\
& +2 i e^{-q \pi i} \sin \{(n+q) \pi\} P_{n}^{(q, p)}\left(x e^{-\pi i}\right)
\end{aligned}
$$

Hence, from this and (1.23), we obtain (assuming temporarily that $n$ is not an integer)

$$
Q_{n}^{(p, q)}\left(-1+(x+1) e^{2 \pi i}\right)=\frac{\pi e^{-(n+p+2 q) \pi i}}{2 \sin (n \pi)}\left[P_{n}^{(p, q)}(x)-e^{-n \pi i} P_{n}^{(q, p)}\left(x e^{-\pi i}\right)\right]
$$

noting that $P_{n}^{(q, p)}\left(x e^{-\pi i}\right)$, being analytic at $x=-1$ for all values of the parameters, is unchanged when $x$ is continued to $-1+(x+1) e^{2 \pi i}$. Thus we arrive at

$$
Q_{n}^{(p, q)}\left(-1+(x+1) e^{2 \pi i}\right)=e^{-2 q \pi i} Q_{n}^{(p, q)}(x)+\pi i e^{-(n+p+2 q) \pi i} P_{n}^{(q, p)}\left(x e^{-\pi i}\right)
$$

where $n$ again can be an integer.

Similarly, if $y\left(1+(x-1) e^{2 \pi i}\right)$ denotes the branch of a solution $y(x)$ obtained by encircling the singularity $x=1$ (but not $x=-1$ ) once in the positive sense, then

$$
Q_{n}^{(p, q)}\left(1+(x-1) e^{2 \pi i}\right)=e^{-2 p \pi i} Q_{n}^{(p, q)}(x)-\pi i e^{-p \pi i} P_{n}^{(p, q)}(x) .
$$

The plan of this paper is as follows. In $\S 2$, we obtain asymptotic approximations for the case where $q$ is large, $p$ is fixed, and $n$ taking any value in the range $0 \leq$ $n \leq O(q)$. We shall show that, for this case, the resulting differential equation has a turning point lying in the interval $(-1,1)$ which is bounded away from a double pole at $x=-1$, but which can coalesce with a simple pole (at $x=1$ ) when $q \rightarrow \infty$ with $n=o(q)$. We apply the theory of [6] which provides asymptotic approximations in terms of Whittaker's confluent hypergeometric functions, which are uniformly valid 
for complex values of $x$, and in particular, in unbounded domains which contain the singularities $x= \pm 1$ as well as the turning point. We obtain approximations for all three Jacobi functions $P_{n}^{(p, q)}(x), P_{n}^{(q, p)}\left(x e^{-\pi i}\right)$, and $Q_{n}^{(p, q)}(x)$ directly, but for the first two only in restricted domains. However, we show how these approximations can be extended to all real or complex $x$ values with the aid of appropriate connection formulas. As we remarked above, Nestor [12] obtained similar approximations for $P_{n}^{(p, q)}(x)$ and $Q_{n}^{(p, q)}(x)$, but only for real $x$ lying in the interval $(1, \infty)$. The results of $\S 2$ are valid for all complex $x$ (with the aid of appropriate connection formulas, if necessary), and in particular, for the special case of $x$ real for $-\infty<x<\infty$.

In $\S 3$, we obtain asymptotic approximations for the case where $n$ is large, $p$ is fixed, and $q$ takes any value in the range $0 \leq q \leq O(n)$. Again we find that the differential equation has a turning point which lies in the interval $(-1,1)$, but this time it is bounded away from the simple pole (at $x=1$ ) but can coalesce with the double pole (at $x=-1$ ) when $n \rightarrow \infty$ with $q=o(n)$. For this case, the theory of [4] is applicable and provides asymptotic expansions in terms of Bessel functions, which are uniformly valid for complex values of $x$ in unbounded domains which contain the double pole $x=-1$ and the coalescing turning point, with only a neighborhood of the simple pole $x=1$ excluded. We obtain expansions for the Jacobi functions $P_{n}^{(q, p)}\left(x e^{-\pi i}\right)$ and $Q_{n}^{(p, q)}(x)$ directly, with the corresponding expansion for $P_{n}^{(p, q)}(x)$ then coming either from (1.11) (if $n \in \mathbf{N}$ ) or from (1.23) otherwise. For approximations which are valid in complex domains containing the simple pole at $x=1$ for the case $n$ large, with $p$ and $q$ fixed (so that there is no turning point), we refer the reader to [7] and [16].

In $\S 4$ and $\S 5$, we consider the case where $p=q$, which corresponds to the equation satistied by the ultraspherical (Gegenbauer) polynomials $C_{n}^{(r)}(x)$ defined by (1.5). In $\S 4$, we consider the case where $p$ and $q$ are large, and the results are uniformly valid for $n$ taking any value (again not necessarily integer values) in the range $0 \leq n \leq O(p)$. For these parameter ranges, we find that the equation has two turning points in $(-1,1)$, which are symmetrically located about the origin and bounded away from poles located at $x= \pm 1$, but which coalesce with one another when $p=q \rightarrow \infty$ with $n=o(p)$. A general asymptotic theory of coalescing turning points is provided by [11], but only for real values of the independent variable. However, since the differential equation in question is even in $x$, we can apply a simple transformation $t=x^{2}$ so that the resulting equation (in terms of $t$ ) is of the type considered in $\S 2$, namely one having a coalescing turning point and simple pole. We, therefore, again are able to apply the theory of [6], and the resulting asymptotic approximations involving Whittaker functions are uniformly valid for complex values of $t$ in unbounded domains which contain the turning point and simple pole. As in $\S 2$, we obtain approximations for all three Jacobi functions $P_{n}^{(p, p)}(x), P_{n}^{(p, p)}\left(x e^{-\pi i}\right)$, and $Q_{n}^{(p, p)}(x)$ directly, but for the first two only in restricted domains. The approximation for $P_{n}^{(p, p)}(x)$ is extended to all real or complex $t$ (and corresponding $x$ values) via a matching with a solution of Jacobi's equation which vanishes at $x=0$.

Finally, in $\S 5$ we consider the case where $n$ is large, and the results given are uniformly valid for $p$ and $q$ taking any value in the range $0 \leq p=q \leq O(n)$. For these parameter ranges we find, as in $\S 4$, that the equation has two turning points in $(-1,1)$ which are symmetrically located about the origin, but this time are bounded away from one another, and which coalesce simultaneously with double poles located at $x= \pm 1$ when $n \rightarrow \infty$ with $p=q=o(n)$. As in $\S 3$ the relevant general asymptotic theory is provided by [4]. We consider the case $|\arg (x)| \leq \frac{1}{2} \pi$ (i.e., focusing on the 
turning point which can coalesce with the double pole at $x=1$ ), with extension to other values of $x$ obtainable from appropriate continuation formulas.

The general theories of [4] and [6] which are applied in this paper provide explicit error bounds for all the approximations.

2. $q$ large, $n$ small or large, and $p$ fixed. For convenience we first define

$$
\tilde{p}=\frac{p}{u}, \quad \tilde{q}=\frac{q}{u}
$$

where $u$ is defined by (1.2). Since all parameters are to be non-negative, we observe from (1.2) and (2.1) that $0 \leq \tilde{p}, \tilde{q}<2$ and $0<u<\infty$. In this section, we suppose that $p$ is fixed (and hence $\tilde{p} \rightarrow 0$ as $u \rightarrow \infty$ ) and allow $q$ to satisfy

$$
\delta \leq \tilde{q}<2
$$

where here and throughout $\delta$ is used generically to denote an arbitrary small positive constant. If $p$ is large and $q$ is fixed, the results of this section are applicable via the relations (1.23) and (1.24) above.

We begin by removing the first derivative in (1.1) by observing that if $y(x)$ is any solution of Jacobi's differential equation, then $w(x)=(x-1)^{(p+1) / 2}(x+1)^{(q+1) / 2} y(x)$ satisfies

$$
w^{\prime \prime}=\left\{u^{2} f(\tilde{q}, x)+g(x)\right\} w
$$

where

$$
\begin{gathered}
g(x)=\frac{p^{2}-1}{2(x-1)^{2}(x+1)}-\frac{1}{4(x+1)^{2}} \\
f(\tilde{q}, x)=\frac{x-x_{t}(\tilde{q})}{(x-1)(x+1)^{2}}
\end{gathered}
$$

and for convenience,

$$
x_{t}(\tilde{q})=\frac{1}{2} \tilde{q}^{2}-1
$$

Eq. (2.3) has a turning point at $x=x_{t}(\tilde{q})$ and regular singularities at $x= \pm 1$. The dominant term, $f(\tilde{q}, x)$, has a simple pole at $x=1$ and a double pole at $x=-1$. From (2.2), we observe that the turning point lies in the interval

$$
-1+\delta \leq x_{t}(\tilde{q})<1,
$$

and as such can be arbitrarily close to the simple pole (which we term as "coalescing"), but is bounded away from the double pole. Note that (2.7) incorporates the case $q \rightarrow \infty$ with $n=o(q)$ since $q^{2} /\left(2 u^{2}\right) \rightarrow 2$ in this case, and consequently from (1.2), $(2.1)$, and (2.6), we see that $x_{t}(\tilde{q}) \rightarrow 1$.

The theory of Dunster [6] is applicable here, and in the notation of that paper $a=2-\frac{1}{2} \tilde{q}^{2}$. Following [6], we apply the following general Liouville transformation (of new independent variable $\xi(x)$ and dependent variable $\hat{W}(\xi)$ )

$$
\hat{W}(\xi)=\left(\frac{d \xi}{d x}\right)^{\frac{1}{2}} w(x),
$$


which takes Eq. (2.3) to the form

$$
\frac{d^{2} \hat{W}}{d \xi^{2}}=\left\{u^{2} \dot{x}^{2} \frac{x-x_{t}(\tilde{q})}{(x-1)(x+1)^{2}}+\dot{x}^{2} g(x)+\dot{x}^{\frac{1}{2}} \frac{d^{2}}{d \xi^{2}}\left(\dot{x}^{-\frac{1}{2}}\right)\right\} \hat{W}
$$

where $\dot{x}=d x / d \xi$. We now choose $\xi(x)$ so that the coefficient of $u^{2}$ is simpler, but retains the characteristic of a simple pole and a coalescing turning point. Thus, for suitably chosen $\alpha=\alpha(u, \tilde{q})$ (see (2.18) below), we prescribe

$$
\dot{x}^{2} \frac{x-x_{t}(\tilde{q})}{(x-1)(x+1)^{2}}=\frac{\xi-\alpha}{\xi} .
$$

Thus, from (2.8),

$$
\hat{W}(\xi)=\left(\frac{x-x_{t}(a)}{\alpha-\xi}\right)^{\frac{1}{4}} \frac{\xi^{\frac{1}{4}}}{(x-1)^{\frac{1}{4}}(x+1)^{\frac{1}{2}}} w(x)
$$

and the result is

$$
\frac{d^{2} \hat{W}}{d \xi^{2}}=\left\{u^{2} \frac{\xi-\alpha}{\xi}+\frac{p^{2}-1}{4 \xi^{2}}+\frac{\hat{\psi}(\alpha, \xi)}{\xi}\right\} \hat{W}
$$

In this equation, $\hat{\psi}(\alpha, \xi)$ is given explicitly by

$$
\begin{aligned}
\hat{\psi}(\alpha, \xi)=\frac{1-p^{2}}{4 \xi} & +\frac{\left(1-p^{2}\right)(\xi-\alpha)(x+1)}{2(1-x)\left(x-x_{t}(\tilde{q})\right)}+\frac{\alpha(8 \xi-3 \alpha)}{16 \xi(\xi-\alpha)^{2}} \\
& +\frac{(\xi-\alpha)(x+1)\left(1-x_{t}(\tilde{q})\right)\left\{4 x^{2}+7 x-5+x_{t}(\tilde{q})(x-7)\right\}}{16(x-1)\left(x-x_{t}(\tilde{q})\right)^{3}}
\end{aligned}
$$

and will be seen to be analytic at $\xi=0$ (which will correspond to $x=1$ ). For suitably chosen $\alpha$, it also will be analytic at $\zeta=\alpha$ (which will correspond to $x=x_{t}(\tilde{q})$ ). Moreover, we shall show that $\xi$ becomes unbounded at both $x=-1$ and $x=\infty$, and that $\hat{\psi}^{\prime}(\alpha, \xi) / \xi$ will be integrable at both these singularities. This in turn will establish that the asymptotic results of this section will be valid at all three singularities of the original equation (1.1).

Let us first suppose that $x$ (and $\xi$ ) are real. Then, integration of relation (2.10) yields for $x_{t}(\tilde{q}) \leq x<1(0<\xi \leq \alpha)$

$$
\int_{0}^{\xi}\left\{\frac{\alpha-\tau}{\tau}\right\}^{\frac{1}{2}} d \tau=\int_{x}^{1}\left\{\frac{t-x_{t}(\tilde{q})}{1-t}\right\}^{\frac{1}{2}} \frac{d t}{t+1}
$$

where the arbitrary integration limits are chosen so that $\xi=0$ corresponds to $x=1$.

Hence, for $x_{t}(\tilde{q}) \leq x<1$,

$$
\begin{aligned}
\sqrt{\xi(\alpha-\xi)}+\frac{\alpha}{2} \arccos \left\{\frac{\alpha-2 \xi}{\alpha}\right\} & =\arccos \left\{\frac{2 x-1-x_{t}(\tilde{q})}{1-x_{t}(\tilde{q})}\right\} \\
- & \left(1-\frac{1}{2} \alpha\right) \arccos \left\{\frac{3 x-1-x_{t}(\tilde{q})(3-x)}{(1+x)\left(1-x_{t}(\tilde{q})\right)}\right\}
\end{aligned}
$$

The branch of the inverse cosines are such that they are continuous and increase montonically from 0 to $\pi$ as $x$ increases from 0 to $\alpha$ (respectively, $x$ decreases from 1 
to $\left.x_{t}(\tilde{q})\right)$. Thus, for the turning points to correspond, i.e., $x=x_{t}(\tilde{q})$ to map to $\xi=\alpha$, we require

$$
\int_{0}^{\alpha}\left\{\frac{\alpha-\tau}{\tau}\right\}^{\frac{1}{2}} d \tau=\int_{x_{t}(\tilde{q})}^{1}\left\{\frac{t-x_{t}(\tilde{q})}{1-t}\right\}^{\frac{1}{2}} \frac{d t}{t+1}
$$

or, equivalently,

$$
\frac{1}{2} \alpha=1-\sqrt{\frac{1}{2}\left(1+x_{t}(\tilde{q})\right)} .
$$

Invoking (2.6) yields

$$
\alpha=2-\tilde{q},
$$

and as such the restriction $\tilde{q} \in[\delta, 2)$ corresponds to

$$
0 \leq \alpha \leq 2-\delta \text {. }
$$

For $-1<x \leq x_{t}(\tilde{q})(\alpha \leq \xi<\infty)$, we have

$$
\int_{\alpha}^{\xi}\left\{\frac{\tau-\alpha}{\tau}\right\}^{\frac{1}{2}} d \tau=\int_{x}^{x_{t}(\tilde{q})}\left\{\frac{x_{t}(\tilde{q})-t}{1-t}\right\}^{\frac{1}{2}} \frac{d t}{t+1}
$$

and hence

$$
\begin{gathered}
{[\xi(\xi-\alpha)]^{\frac{1}{2}}-\frac{1}{2} \alpha \ln \left\{2 \xi-\alpha+2[\xi(\xi-\alpha)]^{\frac{1}{2}}\right\}+\frac{1}{2} \alpha \ln (\alpha)} \\
=\ln \left\{\frac{1-2 x+x_{t}(\tilde{q})-2\left[(1-x)\left(x_{t}(\tilde{q})-x\right)\right]^{\frac{1}{2}}}{1-x_{t}(\tilde{q})}\right\} \\
-\left(1-\frac{1}{2} \alpha\right) \ln \left\{\frac{1-3 x+x_{t}(\tilde{q})(3-x)-2\left[2\left(1+x_{t}(\tilde{q})\right)(1-x)\left(x_{t}(\tilde{q})-x\right)\right]^{\frac{1}{2}}}{(1+x)\left(1-x_{t}(\tilde{q})\right)}\right\} .
\end{gathered}
$$

Finally, for $1<x<\infty(-\infty<\xi<0)$,

$$
\int_{\xi}^{0}\left\{\frac{\tau-\alpha}{\tau}\right\}^{\frac{1}{2}} d \tau=\int_{1}^{x}\left\{\frac{t-x_{t}(\tilde{q})}{t-1}\right\}^{\frac{1}{2}} \frac{d t}{t+1}
$$

and, hence,

$$
\begin{gathered}
{[-\xi(\alpha-\xi)]^{\frac{1}{2}}-\frac{1}{2} \alpha \ln \left\{\alpha-2 \xi-2[-\xi(\alpha-\xi)]^{\frac{1}{2}}\right\}+\frac{1}{2} \alpha \ln (\alpha)} \\
=\ln \left\{\frac{2 x-1-x_{t}(\tilde{q})+2\left[(x-1)\left(x-x_{t}(\tilde{q})\right)\right]^{\frac{1}{2}}}{1-x_{t}(\tilde{q})}\right\} \\
-\left(1-\frac{1}{2} \alpha\right) \ln \left\{\frac{3 x-1+x_{t}(\tilde{q})(x-3)+2\left[2\left(1+x_{t}(\tilde{q})\right)(x-1)\left(x-x_{t}(\tilde{q})\right)\right]^{\frac{1}{2}}}{\left(1-x_{t}(\tilde{q})\right)(x+1)}\right\} .
\end{gathered}
$$

For complex $x$ and $\xi$, the relationship (2.21) relates the two variables. The branches are chosen so that $\xi$ is real and positive for $x$ real and lying in the interval $-1<x<1$, and a continuous function of $x$ elsewhere for $x$ lying in the plane having a branch cut along the real axis from $x=-1$ to $x=-\infty$. 


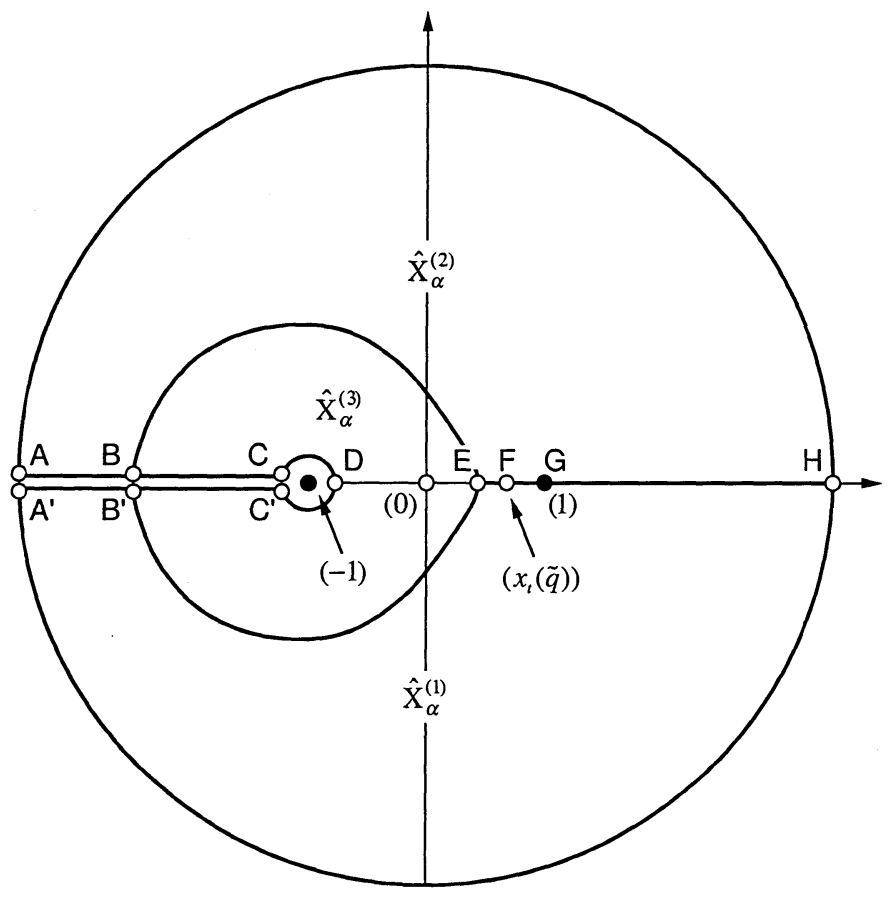

Figure 1(a): $x$ plane

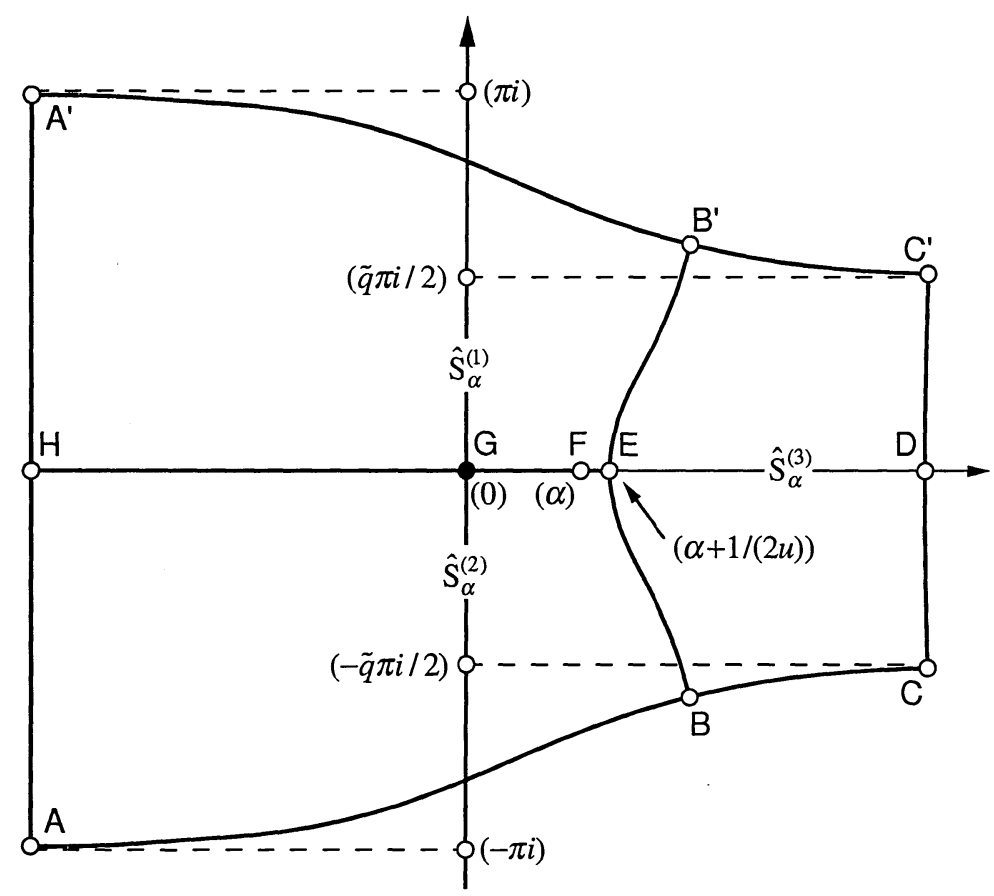

Figure 1(b): $\xi$ plane 
Figure 1(b) shows the $\xi$ domain corresponding to the cut $x$ domain which is depicted in Figure 1(a) (with neighborhoods of the singularities $x=-1$ and $x=\infty$ excluded). In Figure 1(b), the curve $A C$ in the $\xi$ plane corresponds to the upper lip of the cut from $x=-1$ to $x=-\infty$ and is given parametrically by

$$
\int_{\alpha}^{\xi}\left\{\frac{\tau-\alpha}{\tau}\right\}^{\frac{1}{2}} d \tau=s-\left(1-\frac{1}{2} \alpha\right) \pi i \quad(-\infty<s<\infty) .
$$

This curve has horizontal asymptotes of $\operatorname{Im} \xi=-\pi i$ and $\operatorname{Im} \xi=-\left(1-\frac{1}{2} \alpha\right) \pi i=-\frac{1}{2} \tilde{q} \pi i$ at $\operatorname{Re} \xi=-\infty$ and $\operatorname{Re} \xi=\infty$, respectively. The curve $A^{\prime} C^{\prime}$, corresponding to the lower lip of the cut from $x=-1$ to $x=-\infty$, is the conjugate of $A C$.

Also, in Figure 1(b), three subdomains $\hat{S}_{\alpha}^{(j)}, j=1,2,3$, of the $x$ plane are depicted (see $[6, \S 4])$. With respect to the singularity at $\xi=0$, the values of $\arg (\xi)$ in each of these three domains will be chosen according to the solution being examined (see below). We remark that the domain $\hat{S}_{\alpha}^{(3)}$ is the one in which $P_{n}^{(q, p)}\left(x e^{-\pi i}\right)$ is recessive (for large $u$ and considered as a function of $x$ ). The curve $B E B^{\prime}$ which, along with the interval $(-\infty, \alpha+1 /(2 u)]$, separates the three domains is given by

$$
\operatorname{Re} \int_{\alpha+1 /(2 u)}^{\xi}\left(\frac{\tau-\alpha}{\tau}\right)^{\frac{1}{2}} d \tau=0
$$

and emanates from $\xi=\alpha+1 /(2 u)$ at an angle of $\frac{1}{2} \pi$ with the positive real axis. If this curve were to be continued to infinity, it would be asymptotic to the curve (when $\alpha>0)$

$$
(\operatorname{Im} \xi)^{2}=\frac{1}{16 u^{2}}(1+b)^{2} \exp \left\{\frac{2}{u \alpha}(2 u \operatorname{Re} \xi-b)\right\}-(\operatorname{Re} \xi)^{2}
$$

where

$$
b=u \alpha+\sqrt{2 u \alpha+1}
$$

When $\alpha=0$, the curve becomes the vertical line $\operatorname{Re} \xi=1 /(2 u)$. The corresponding curve in the $x$ plane envelops the singularity at $x=-1$, as shown in Figure 1(a), and in this figure, the three $x$ domains corresponding to $\hat{S}_{\alpha}^{(j)}, j=1,2,3$, are denoted by $\hat{X}_{\alpha}^{(j)}, j=1,2,3$, respectively.

We shall choose the arguments of both variables according to the solution that we are studying. For the function $Q_{n}^{(p, q)}(x)$, we introduce a cut along the real axis from $x=1$ to $x=-\infty$ and, correspondingly, from $\zeta=0$ to $\zeta=\infty$. Then in these cut domains, we specify that $0 \leq \arg (\zeta)<2 \pi$ and $-\pi<\arg (x-1) \leq \pi$. For $P_{n}^{(q, p)}\left(x e^{-\pi i}\right)$, we introduce a cut along the real axis from $x=1$ to $x=\infty$ and, correspondingly, from $\zeta=0$ to $\zeta=-\infty$. Then in these cut domains, we specify that $-\pi<\arg (\zeta) \leq \pi$ and $0 \leq \arg (x-1)<2 \pi$. For $P_{n}^{(p, q)}(x)$, no further cut is required since this function is analytic at $x=1$. However, with regard to the cut from $x=-1$ to $x=-\infty$, we specify that $-\pi<\arg (x+1) \leq \pi$, and in the corresponding $\zeta$ plane, $\arg (\zeta)$ takes its principal value.

We next record the behavior of the two variables in the vicinity of the three singularities. Firstly, from (2.23), we find that $\xi \rightarrow 0$ as $x \rightarrow 1$ such that

$$
x=1-\frac{8}{4-\alpha} \xi+\frac{8(24-7 \alpha)}{3(4-\alpha)^{3}} \xi^{2}+O\left(\xi^{3}\right) .
$$


Thus, from (2.13), we find that

$$
\hat{\psi}(\alpha, \xi)=\frac{\alpha\left(p^{2}-1\right)}{6(4-\alpha)^{2}}+O(\xi), \quad \xi \rightarrow 0 .
$$

Next, from (2.23), we see that $\operatorname{Re} \xi \rightarrow-\infty$ as $x \rightarrow \infty$ such that

$$
\begin{aligned}
-\xi+\frac{1}{2} \alpha \ln (-\xi)+ & \frac{1}{2} \alpha+\frac{1}{2} \alpha \ln \left(\frac{4}{\alpha}\right)+O\left(\xi^{-1}\right)=\ln (x)-\ln \left\{1-x_{t}(\tilde{q})\right\} \\
& -\left(1-\frac{1}{2} \alpha\right) \ln \left\{\frac{3+x_{t}(\tilde{q})+\sqrt{2\left(1+x_{t}(\tilde{q})\right)}}{1-x_{t}(\tilde{q})}\right\}+O\left(x^{-1}\right)
\end{aligned}
$$

which on exponentiating and invoking (2.17) yields

$$
x=2 \exp \left\{\frac{1}{2} \alpha+\left(2-\frac{1}{2} \alpha\right) \ln \left(1-\frac{1}{4} \alpha\right)\right\}(-\xi)^{\frac{1}{2} \alpha} e^{-\xi}\left\{1+O\left(\xi^{-1}\right)\right\}
$$

as $\operatorname{Re} \xi \rightarrow-\infty$.

Finally, from (2.21), we find that $\operatorname{Re} \xi \rightarrow \infty$ as $x \rightarrow-1^{+}$such that

$$
\begin{aligned}
\xi-\frac{1}{2} \alpha \ln (\xi)-\frac{1}{2} \alpha+\frac{1}{2} \alpha \ln \left(\frac{1}{4} \alpha\right) & +O\left(\xi^{-1}\right)=\left(1-\frac{1}{2} \alpha\right) \ln \left\{\frac{1+x_{t}(\tilde{q})}{8\left(1-x_{t}(\tilde{q})\right)(1+x)}\right\} \\
+ & \ln \left\{\frac{3+x_{t}(\tilde{q})-2 \sqrt{2\left(1+x_{t}(\tilde{q})\right)}}{1-x_{t}(\tilde{q})}\right\}+O(x+1)
\end{aligned}
$$

and hence, again on exponentiating,

$$
\begin{aligned}
x=-1+(2- & \alpha)^{2} e^{-2 \xi /(2-\alpha)} \xi^{\alpha /(2-\alpha)} \\
& \times \exp \left\{\frac{\alpha+(6-\alpha) \ln (2)-(4-\alpha) \ln (4-\alpha)}{2-\alpha}\right\}\left\{1+O\left(\xi^{-1}\right)\right\} .
\end{aligned}
$$

Thus, from (2.13), (2.31), and (2.33), we see that, as $\xi \rightarrow \infty$,

$$
\hat{\psi}(\alpha, \xi)=\frac{1-p^{2}}{4 \xi}+O\left(\frac{1}{\xi^{2}}\right)
$$

regardless of the direction at which $\xi$ approaches infinity, i.e., (2.34) holds as $x \rightarrow \infty$ or as $x \rightarrow-1$. From the error bounds supplied by [6, §4] this confirms that our approximations will hold at all three singularities of Jacobi's equation. If we neglect the $\hat{\psi}\left(a^{\prime}, \xi\right) / \xi$ term in Eq. (2.12), we have the so-called comparison equation

$$
\frac{d^{2} \hat{W}}{d \xi^{2}}=\left\{u^{2} \frac{\xi-\alpha}{\xi}+\frac{p^{2}-1}{4 \xi^{2}}\right\} \hat{W}
$$

which has exact solutions in terms of the Whittaker's confluent hypergeometric functions $M_{\frac{1}{2} u \alpha, \frac{1}{2} p}(2 u \xi), W_{\frac{1}{2} u \alpha, \frac{1}{2} p}(2 u \xi)$ and $W_{-\frac{1}{2} u \alpha, \frac{1}{2} p}\left(2 u \xi e^{-\pi i}\right)$. Recall that $p$ is fixed, so these are functions of two free variables, and therefore, if they are used as approximants, they achieve a uniform reduction of free variables. In [6], it was proved that these Whittaker functions are indeed asymptotic approximations for solutions of 
(2.12), and in particular, Theorem 2 of [6] provides the following solutions of (2.12) which are recessive at certain singularities of the $\xi$ plane

$$
\hat{W}^{(j)}(u, \alpha, \xi)=\hat{\mathcal{U}}_{\frac{1}{2} u \alpha, \frac{1}{2} p}^{(j)}(2 u \xi)+\hat{\varepsilon}^{(j)}(u, \alpha, \xi)
$$

where

$$
\begin{aligned}
& \hat{\mathcal{U}}_{k, m}^{(0)}(z)=\frac{\Gamma\left(k+m+\frac{1}{2}\right)}{\gamma(k) \Gamma(1+2 m)} M_{k, m}(z), \\
& \hat{\mathcal{U}}_{k, m}^{(1)}(z)=e^{-m \pi i} \gamma(k) W_{-k, m}\left(z e^{-\pi i}\right), \\
& \hat{\mathcal{U}}_{k, m}^{(4)}(z)=\frac{e^{\left(k-m-\frac{1}{2}\right) \pi i}}{\gamma(k)} W_{k, m}(z),
\end{aligned}
$$

with

$$
\gamma(k)=k^{k} e^{-k}
$$

Theorem 2 of [6] provides explicit bounds for the error terms $\hat{\varepsilon}^{(j)}(u, \alpha, \xi)$ appearing in the asymptotic solutions (2.36), which are uniformly valid in certain unbounded complex domains containing the pole and turning point, and for $\alpha \in[0,2-\delta]$ in the present case.

Let us consider $j=0$ first. It is well known that

$$
M_{k, m}(z) \sim z^{m+\frac{1}{2}}, \quad z \rightarrow 0,-2 m \notin \mathbf{N},
$$

and hence, $\hat{W}^{(0)}(u, \alpha, \xi)$ is uniquely characterized as being recessive at the singularity $\xi=0$ (i.e., $x=1$ ). The bound on $\hat{\varepsilon}^{(0)}(u, \alpha, \xi)$ establishes that, except near the zeros of $\hat{\mathcal{U}}_{\frac{1}{2} u \alpha, \frac{1}{2} p}^{(0)}(2 u \xi)$,

$$
\hat{\varepsilon}^{(0)}(u, \alpha, \xi)=\hat{\mathcal{U}}_{\frac{1}{2} u \alpha, \frac{1}{2} p}^{(0)}(2 u \xi) O\left\{(u \alpha+1)^{\frac{1}{3}} u^{-1+\delta}\right\}
$$

uniformly for all $\xi$ under consideration when $n \notin \mathbf{N}$, and in $\xi \in \hat{S}_{\alpha}^{(1)} \cup \hat{S}_{\alpha}^{(2)}$ otherwise (see [6, Eq. (4.66)]). It is worth remarking that when $n \in \mathrm{N}$, the region $\hat{S}_{\alpha}^{(3)}$ must be excluded because in this case the approximant $\hat{\mathcal{U}}_{\frac{1}{2} u \alpha, \frac{1}{2} p}^{(0)}(2 u \xi)$ is recessive at both $\xi=0$ and at infinity in $\hat{S}_{\alpha}^{(3)}$ (i.e., at $x= \pm 1$ ); in essence the region of validity is the union of a neighborhood of $\xi=0$ (the characterizing singularity of recessiveness of $\left.\hat{\mathcal{U}}_{\frac{1}{2} u \alpha, \frac{1}{2} p}^{(0)}(2 u \xi)\right)$ and adjacent regions in which $\hat{\mathcal{U}}_{\frac{1}{2} u \alpha, \frac{1}{2} p}^{(0)}(2 u \xi)$ is dominant. Therefore, since $\hat{\mathcal{U}}_{\frac{1}{2} u \alpha, \frac{1}{2} p}^{(0)}(2 u \xi)$ is recessive in $\hat{S}_{\alpha}^{(3)}$ when $n \in \mathrm{N}$, this domain must be excluded from the region of asymptotic validity.

We are now in a position to make an identification of the asymptotic solution (2.36) when $j=0$ with a standard solution of Jacobi's equation. In particular, we can assert the existence of a constant $\hat{D}^{(0)}(u, \alpha)$ such that

$$
P_{n}^{(p, q)}(x)=\frac{\hat{D}^{(0)}(u, \alpha)}{(1-x)^{\frac{1}{2} p+\frac{1}{4}}(1+x)^{\frac{1}{2} q}}\left\{\frac{\alpha-\xi}{\xi\left(x-x_{t}(a)\right)}\right\}^{\frac{1}{4}}\left\{\hat{\mathcal{U}}_{\frac{1}{2} u \alpha, \frac{1}{2} p}^{(0)}(2 u \xi)+\hat{\varepsilon}^{(0)}(u, \alpha, \xi)\right\}
$$


since both sides of (2.43) are solutions of Eq. (2.12) which are recessive at $x=1$ $(\xi=0)$. Using (1.12), (2.28), (2.37), (2.40) - (2.42), we can compare the behavior of both solutions at $x=1$, and from this, we find that

$$
\hat{D}^{(0)}(u, \alpha)=\frac{2^{p+q-u} e^{\frac{1}{2} q-u}(2 u+q)^{-\frac{1}{2} p}(2 u-q)^{u-\frac{1}{2} q}}{\sqrt{u} \Gamma(n+1)} .
$$

Nex.t, for some constant $\hat{D}^{(4)}(u, \alpha)$, we make the following identification of two solutions which are recessive at $x=-1(\xi=+\infty)$

$$
\begin{aligned}
P_{n}^{(q, p)}\left(x e^{-\pi i}\right)=\frac{\hat{D}^{(4)}(u, \alpha)}{(1-x)^{\frac{1}{2} p+\frac{1}{4}}(1+x)^{\frac{1}{2} q}}\{ & \left.\frac{\alpha-\xi}{\xi\left(x-x_{t}(a)\right)}\right\}^{\frac{1}{4}} \\
& \times\left\{\hat{\mathcal{U}}_{\frac{1}{2} u \alpha, \frac{1}{2} p}^{(4)}(2 u \xi)+\hat{\varepsilon}^{(4)}(u, \alpha, \xi)\right\}
\end{aligned}
$$

By comparing both sides as $x \rightarrow-1^{+}$and invoking (1.12) (with $p$ and $q$ interchanged and $x$ replaced by $-x),(2.32)$, and [6, Eq. (4.24)], we arrive at

$$
\hat{D}^{(4)}(u, \alpha)=e^{-n \pi i} \frac{2^{\frac{1}{2}(p+3 q)} q^{q+\frac{1}{2}}(2 u-q)^{u-\frac{1}{2} q} \Gamma(n+q+1)}{\sqrt{u}(2 u+q)^{u+\frac{1}{2} q} \Gamma(n+1) \Gamma(q+1)} .
$$

Eq. (2.45) then provides a uniform asymptotic approximation which is valid for $\xi \in \hat{S}_{\alpha}^{(3)}$ (but not for $\xi \in \hat{S}_{\alpha}^{(1)} \cup \hat{S}_{\alpha}^{(2)}$ ).

Next, we have the relationship

$$
Q_{n}^{(p, q)}(x)=\frac{\hat{D}^{(1)}(u, \alpha)}{(x-1)^{\frac{1}{2} p+\frac{1}{4}(x+1)^{\frac{1}{2} q}}}\left\{\frac{\xi-\alpha}{\xi\left(x-x_{t}(a)\right)}\right\}^{\frac{1}{4}}\left\{\hat{\mathcal{U}}_{\frac{1}{2} u \alpha, \frac{1}{2} p}^{(1)}(2 u \xi)+\hat{\varepsilon}^{(1)}(u, \alpha, \xi)\right\}
$$

since both functions are solutions of the same equation which are recessive at $x=\infty$. On letting $x \rightarrow \infty(\xi \rightarrow-\infty, \arg (\xi)=\pi)$, we find, from (1.21), (2.31), and [6, Eq. (4.21)], that

$$
\hat{D}^{(1)}(u, \alpha)=e^{\frac{1}{2} p \pi i} K_{n}^{(p, q)} 2^{5 u-n} u^{2 u}(2 u-q)^{\frac{1}{2} q-u}(2 u+q)^{-\frac{1}{2} q-u}
$$

where $K_{n}^{(p, q)}$ is defined by (1.10). This approximation is uniformly valid for all $\xi$ in the domain depicted in Figure 1(b) where $0 \leq \arg (\xi) \leq 2 \pi$ (i.e., for all values of $x$ in the principal plane of $\left.Q_{n}^{(p, q)}(x)\right)$.

In summary, (2.43), (2.45), and (2.47) provide asymptotic approximations for the three standard solutions of Jacobi's equation as $u=n+\frac{1}{2}(p+q+1) \rightarrow \infty$, which are uniformly valid in the stated unbounded complex domains. Although the approximations (2.43) and (2.45) do not hold in the full planes of the respective principal branches, the required extension is easily achieved via appropriate connection formulas. In particular, when $n \in \mathbf{N}$, the approximation (2.43) does not hold for $\xi \in \hat{S}_{\alpha}^{(3)}$ (since $F_{n}^{(p, q)}(x)$ is recessive in this domain under these circumstances), but in this case, we can simply use (1.11) and (2.45) to obtain the required approximation which is valid in $\hat{S}_{\alpha}^{(3)}$. Likewise, when $n \notin \mathbf{N}$, an approximation for $P_{n}^{(q, p)}\left(x e^{-\pi i}\right)$ which is valid for $\xi \in \hat{S}_{\alpha}^{(1)} \cup \hat{S}_{\alpha}^{(2)}$ is obtainable from (1.23), (2.43), and (2.47). Thus, taken together, and with the aid of appropriate connection formulas if needed, the results 
of this section are valid for all values of $x$ in the planes of the principal branches for the three Jacobi functions $P_{n}^{(p, q)}(x), P_{n}^{(q, p)}\left(x e^{-\pi i}\right)$, and $Q_{n}^{(p, q)}(x)$.

The results of this section hold for $q$ large, $p$ fixed, and $n$ small or large, under the restriction (2.2), which is equivalent to

$$
0<\frac{n}{q}+\frac{p+1}{2 q} \leq \frac{1}{\delta}-\frac{1}{2}
$$

Therefore, since $p$ is fixed, for large $q$, the parameter $n$, which does not necessarily have to be an integer, is permitted to take any value in the range

$$
0 \leq n \leq A q
$$

where $A$ is any fixed positive constant (independent of $q$ ).

3. $n$ large, $q$ small or large, and $p$ fixed. We now consider the case where the turning point can coalesce with the double pole, i.e., $-1 \leq x_{t}(\tilde{q}) \leq 1-\delta$. Thus, with the assumption that $p$ is fixed, from the definition (2.6) of $x_{t}(\tilde{q})$, we see that $\tilde{q}$ now will be allowed to lie in the interval $[0,2-\delta]$. Hence from the definitions (1.2) and (2.1), this is seen to be equivalent to

$$
0 \leq q \leq A n
$$

where $A$ is any fixed positive constant (independent of $n$ ). Thus the results of this section will be valid for $p$ fixed, $n$ large, and all $q$ lying in the (large) interval (3.1). The singularities $x=-1$ and $x=\infty$ will be included in the domain of validity, but the singularity $x=1$ must be excluded this time.

From the definition (2.6), we see that the turning point coalesces with the double pole when $\tilde{q} \rightarrow 0$. We shall use the general asymptotic theory of Boyd and Dunster [4] which is applicable to differential equations having a coalescing turning point and double pole in the complex plane. The approximations are asymptotic expansions, and involve Bessel functions.

Firstly, from (2.5), we see that

$$
f(\tilde{q}, x)=\frac{\tilde{q}^{2}}{4(x+1)^{2}}+O\left(\frac{1}{x+1}\right) .
$$

In [4], it was assumed that if the double pole is located at $x=x_{0}$, say, then the leading term of the Laurent expansion of $f$ and $g$ in the vicinity of the double pole (at $x_{0}=-1$ in our case) must be $\frac{1}{4} \alpha^{2}\left(x-x_{0}\right)^{-2}$ and $-\frac{1}{4}\left(x-x_{0}\right)^{-2}$, respectively. Thus, in the notation of [4], $\alpha=\tilde{q}$, and from (2.4), we see that $g$ has the appropriate behavior at $x=-1$.

Now, from [4, Eq. (2.1)], we perceive that the required Liouville transformation is given by

$$
W(\zeta)=\left(\frac{x-x_{t}(\tilde{q})}{\zeta-\tilde{q}^{2}}\right)^{\frac{1}{4}} \frac{\zeta^{\frac{1}{2}}}{(x-1)^{\frac{1}{4}}(x+1)^{\frac{1}{2}}} w(x)
$$

with

$$
\frac{d \zeta}{d x}=\frac{2 \zeta}{x+1}\left\{\frac{x-x_{t}(\tilde{q})}{\left(\zeta-\tilde{q}^{2}\right)(1-x)}\right\}^{\frac{1}{2}}
$$


The result of this is to transform (2.3) into the form

$$
\frac{d^{2} W}{d \zeta^{2}}=\left\{u^{2}\left(\frac{\tilde{q}^{2}}{4 \zeta^{2}}-\frac{1}{4 \zeta}\right)+\frac{\psi(\tilde{q}, \zeta)}{\zeta}-\frac{1}{4 \zeta^{2}}\right\} W
$$

where in this case the Schwarzian is given by

$$
\begin{array}{r}
\psi(\tilde{q}, \zeta)=\frac{\zeta+4 \tilde{q}^{2}}{16\left(\zeta-\tilde{q}^{2}\right)^{2}}+\frac{\zeta-\tilde{q}^{2}}{64 \zeta}\left[\frac{(x+1)\left(1-x_{t}(\tilde{q})\right)\left(4 x^{2}+7 x+x_{t}(\tilde{q})(x-7)-5\right)}{(1-x)\left(x-x_{t}(\tilde{q})\right)^{3}}\right. \\
\left.+\frac{8\left(p^{2}-1\right)(x+1)}{(1-x)\left(x-x_{t}(\tilde{q})\right)}\right]
\end{array}
$$

For large $u$, the new equation (3.5) has a double pole at $\zeta=0$ and a turning point at $\zeta=\tilde{q}$, which will correspond to $x=-1$ and $x=x_{t}(\tilde{q})$, respectively. The asymptotic expansions for solutions of (3.5) furnished by [4] will be valid in certain unbounded domains which will include both of these critical points. We emphasize that the results will be uniformly valid for $0 \leq \tilde{q} \leq 2-\delta$, and hence the turning point is permitted to coalesce with the pole.

Let us first consider real values of the independent variables. For $x_{t}(\tilde{q}) \leq x \leq 1$, integration of (3.4) yields (cf. [4, Eq. (2.2a)])

$$
\int_{\tilde{q}^{2}}^{\zeta} \frac{\left(\tau-\tilde{q}^{2}\right)^{\frac{1}{2}}}{2 \tau} d \tau=\int_{x_{t}(\tilde{q})}^{x}\left\{\frac{t-x_{t}(\tilde{q})}{1-t}\right\}^{\frac{1}{2}} \frac{d t}{t+1}
$$

where the lower limits were chosen for the turning points to correspond. On explicit integration, (3.7) gives the relationship

$$
\begin{aligned}
\sqrt{\zeta-\tilde{q}^{2}}-\tilde{q} \arctan \left\{\frac{\sqrt{\zeta-\tilde{q}^{2}}}{\tilde{q}}\right\}=\arccos & \left\{\frac{x_{t}(\tilde{q})+1-2 x}{1-x_{t}(\tilde{q})}\right\} \\
& -\frac{1}{2} \tilde{q} \arccos \left\{\frac{1-3 x+x_{t}(\tilde{q})(3-x)}{(x+1)\left(1-x_{t}(\tilde{q})\right)}\right\} .
\end{aligned}
$$

The branch of inverse tangent is such that it is continuous and increases montonically from 0 as $\zeta$ increases from $\tilde{q}^{2}$. The branches of inverse cosines are such that they are continuous and increase montonically from 0 to $\pi$ as $x$ increases from $x_{t}(\tilde{q})$ to 1 . From (3.8), we observe that the simple pole at $x=1$ corresponds to the finite point $\zeta=\zeta_{1}$, say, where

$$
\left(\zeta_{1}-\tilde{q}^{2}\right)^{\frac{1}{2}}-\tilde{q} \arctan \left\{\frac{1}{\tilde{q}}\left(\zeta_{1}-\tilde{q}^{2}\right)^{\frac{1}{2}}\right\}=\left(1-\frac{1}{2} \tilde{q}\right) \pi,
$$

when $\tilde{q} \neq 0$. When $\tilde{q}=0(\alpha=2)$, this singularity is located at $\zeta_{1}=\pi^{2}$. In this section, the asymptotic expansions will not be valid in a neighborhood of this singularity.

Next, for $-1<x \leq x_{t}(\tilde{q})$ (or correspondingly, $0<\zeta \leq \tilde{q}$ ),

$$
\int_{\zeta}^{\tilde{q}^{2}} \frac{\left(\tilde{q}^{2}-\tau\right)^{\frac{1}{2}}}{2 \tau} d \tau=\int_{x}^{x_{t}(\tilde{q})}\left\{\frac{x_{t}(\tilde{q})-t}{1-t}\right\}^{\frac{1}{2}} \frac{d t}{t+1}
$$

which on integration yields 


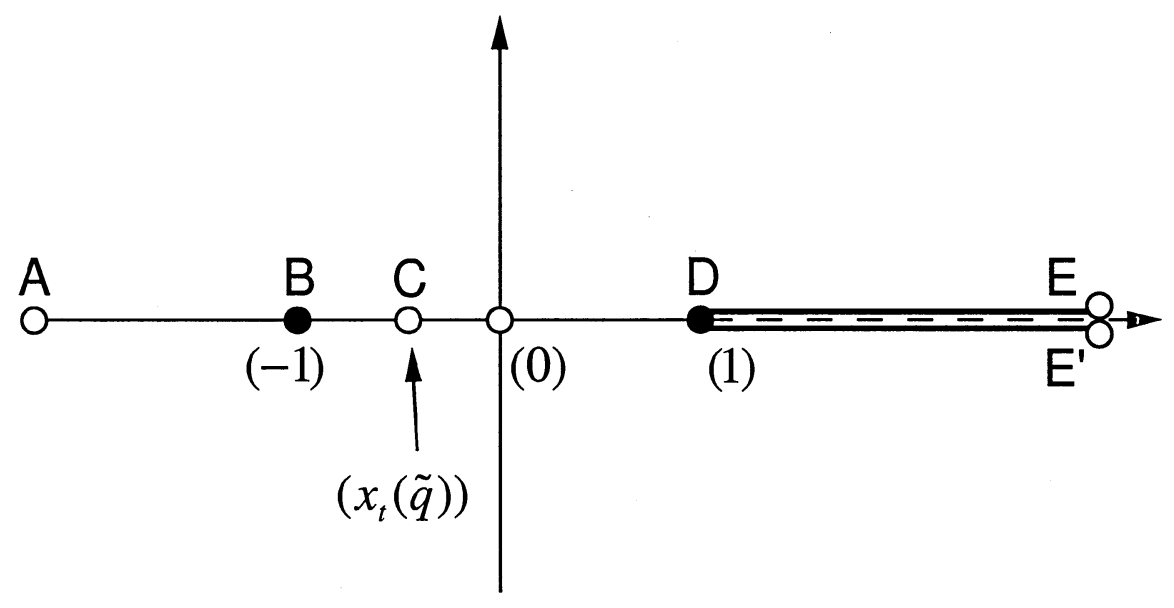

Figure 2(a): $x$ plane

$$
\begin{gathered}
\tilde{q} \ln \left\{\left(\tilde{q}^{2}-\zeta\right)^{\frac{1}{2}}+\tilde{q}\right\}-\frac{1}{2} \tilde{q} \ln (\zeta)-\left(\tilde{q}^{2}-\zeta\right)^{\frac{1}{2}} \\
=\ln \left\{\frac{1-2 x+x_{t}(\tilde{q})-2\left\{(1-x)\left(x_{t}(\tilde{q})-x\right)\right\}^{\frac{1}{2}}}{1-x_{t}(\tilde{q})}\right\} \\
-\frac{1}{2} \tilde{q} \ln \left\{\frac{1-3 x+x_{t}(\tilde{q})(3-x)-2\left\{2\left(1+x_{t}(\tilde{q})\right)(1-x)\left(x_{t}(\tilde{q})-x\right)\right\}^{\frac{1}{2}}}{(x+1)\left(1-x_{t}(\tilde{q})\right)}\right\}
\end{gathered}
$$

From this, we find that $\zeta \rightarrow 0$ as $x \rightarrow-1$ such that

$$
x=-1+\frac{2 e^{2}}{4-\tilde{q}^{2}}\left(\frac{2-\tilde{q}}{2+\tilde{q}}\right)^{\frac{2}{\tilde{q}}} \zeta+O\left(\zeta^{2}\right) .
$$

Finally, for $-\infty<x<-1(-\infty<\zeta<0)$, the relationship is found to be

$$
\begin{aligned}
& \tilde{q} \ln \left\{\left(\tilde{q}^{2}-\zeta\right)^{\frac{1}{2}}+\tilde{q}\right\}-\frac{1}{2} \tilde{q} \ln (-\zeta)-\left(\tilde{q}^{2}-\zeta\right)^{\frac{1}{2}} \\
& \quad=\ln \left\{\frac{1-2 x+x_{t}(\tilde{q})-2\left\{(1-x)\left(x_{t}(\tilde{q})-x\right)\right\}^{\frac{1}{2}}}{1-x_{t}(\tilde{q})}\right\} \\
& \quad-\frac{1}{2} \tilde{q} \ln \left\{\frac{1-3 x+x_{t}(\tilde{q})(3-x)-2\left\{2\left(1+x_{t}(\tilde{q})\right)(1-x)\left(x_{t}(\tilde{q})-x\right)\right\}^{\frac{1}{2}}}{(-x-1)\left(1-x_{t}(\tilde{q})\right)}\right\}
\end{aligned}
$$

For complex values of the variables, we shall introduce a cut along the real axis from $x=-1$ to $x=+\infty$ (and correspondingly $\zeta=0$ to $\zeta=+\infty$ ) and restrict the arguments so that $0 \leq \arg (x+1) \leq 2 \pi$, and correspondingly, $0 \leq \arg (\zeta) \leq 2 \pi$. Then in these cut planes, (3.13) defines the relationship between the variables, where the branches of both sides are selected so that $\zeta(x)$ is real and negative when $x$ is real and lies in the interval $(-\infty,-1)(\arg (x)=\pi)$, and a continuous function of $x$ elsewhere in the cut plane.

Figure 2(b) depicts the $\zeta$ domain, $\Delta$ say, corresponding to the cut $x$ plane (in which $0 \leq \arg (x-1) \leq 2 \pi)$, the latter being shown in Figure $2(\mathrm{a})$. The curves $D E$ and its conjugate $D E^{\prime}$ in Figure 2(b) correspond, respectively, to the upper and lower 


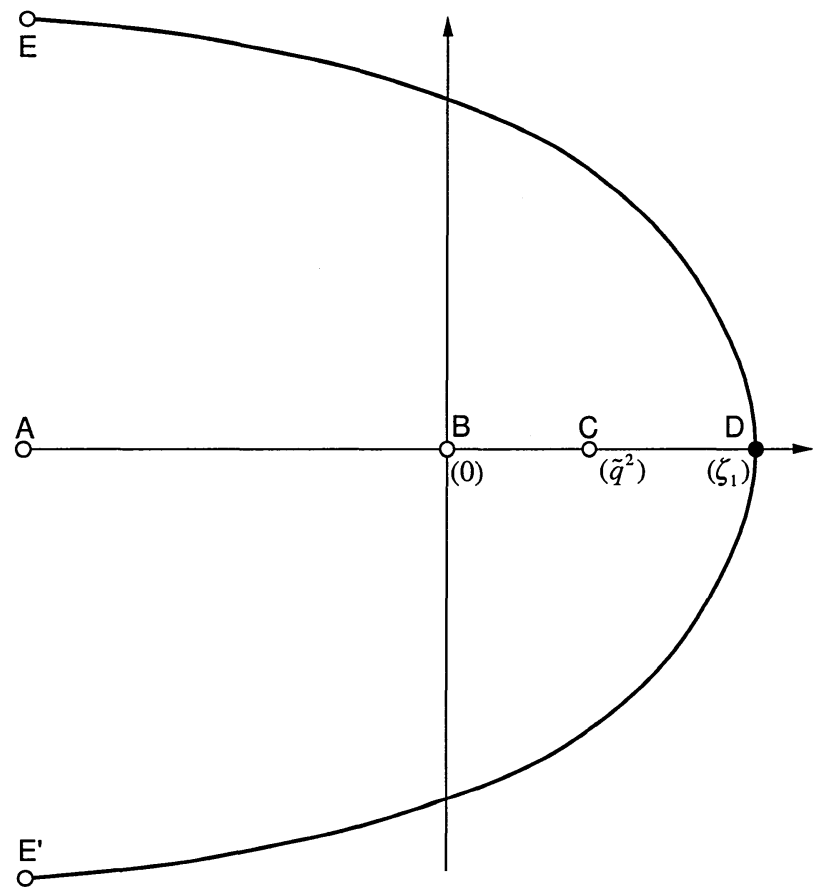

Figure 2(b): $\zeta$ plane

lips of the cut from $x=1$ to $x=\infty$. These curves are given parametrically by

$$
\int_{\zeta_{1}}^{\zeta} \frac{\left(\tau-\tilde{q}^{2}\right)^{\frac{1}{2}}}{2 \tau} d \tau= \pm i s, \quad 0 \leq s<\infty
$$

and at infinity are asymptotic to the parabola $\operatorname{Re} \zeta=\pi^{2}-(\operatorname{Im} \zeta)^{2} /\left(4 \pi^{2}\right)$. Note that $\arg (x-1)=0$ on the upper lip $D E$ of the cut from $x=1$ to $x=\infty$, and hence both $P_{n}^{(p, q)}(x)$ and $Q_{n}^{(p, q)}(x)$ are real on $D E$.

With the above choices of branches, we find, from (3.13), that $\zeta \rightarrow-\infty$ as $x \rightarrow-\infty$ such that

$$
x \sim-\frac{4-\tilde{q}^{2}}{8}\left(\frac{2+\tilde{q}}{2-\tilde{q}}\right)^{\frac{\tilde{q}}{2}} \exp \left\{(-\zeta)^{\frac{1}{2}}\right\}
$$

Having made the appropriate transformation, we apply Theorem 3 of [4] to obtain the following two asymptotic solutions of (3.5)

$$
\begin{aligned}
W_{2 N+1}^{(0)}(u, \tilde{q}, \zeta)=2 \zeta^{\frac{1}{2}} J_{q}\left(u \zeta^{\frac{1}{2}}\right) & \sum_{s=0}^{N} \frac{A_{s}(\tilde{q}, \zeta)}{u^{2 s}} \\
& +\frac{2 \zeta}{u} J_{q}^{\prime}\left(u \zeta^{\frac{1}{2}}\right) \sum_{s=0}^{N-1} \frac{B_{s}(\tilde{q}, \zeta)}{u^{2 s}}+\varepsilon_{2 N+1}^{(0)}(u, \tilde{q}, \zeta),
\end{aligned}
$$

and 


$$
\begin{aligned}
W_{2 N+1}^{(1)}(u, \tilde{q}, \zeta)=\zeta^{\frac{1}{2}} H_{q}^{(1)}\left(u \zeta^{\frac{1}{2}}\right) & \sum_{s=0}^{N} \frac{A_{s}(\tilde{q}, \zeta)}{u^{2 s}} \\
& +\frac{\zeta}{u} H_{q}^{(1)^{\prime}}\left(u \zeta^{\frac{1}{2}}\right) \sum_{s=0}^{N-1} \frac{B_{s}(\tilde{q}, \zeta)}{u^{2 s}}+\varepsilon_{2 N+1}^{(1)}(u, \tilde{q}, \zeta)
\end{aligned}
$$

where $A_{0}(\tilde{q}, \zeta)=1$, and for $s=1,2,3, \ldots$,

$$
B_{s}(\tilde{q}, \zeta)=\left(\zeta-\tilde{q}^{2}\right)^{-\frac{1}{2}} \int_{\tilde{q}^{2}}^{\zeta}\left(\tau-\tilde{q}^{2}\right)^{-\frac{1}{2}}\left\{\tau A_{s}^{\prime \prime}(\tilde{q}, \tau)+A_{s}^{\prime}(\tilde{q}, \tau)-\psi(\tilde{q}, \tau) A_{s}(\tilde{q}, \tau)\right\} d \tau
$$

and

$$
A_{s}(\tilde{q}, \zeta)=-\zeta B_{s-1}^{\prime}(\tilde{q}, \zeta)+\int_{0}^{\zeta} \psi(\tilde{q}, \tau) B_{s-1}(\tilde{q}, \tau) d \tau+\lambda_{s}(\tilde{q})
$$

Here $\left\{\lambda_{s}(\tilde{q})\right\}_{s=1}^{N}$ are arbitrary integration constants, and we shall choose them for our convenience later. The error terms in (3.16) and (3.17) are bounded by Theorem 3 of [4] (with $n$ replaced by $N$ and $\alpha$ replaced by $\tilde{q}$ ). These bounds are valid for all $\zeta \in \Delta$ except for a neighborhood of the singularity at $\zeta=\zeta_{1}$ and prove that both error terms are $\zeta^{\frac{1}{2}} \mathcal{C}_{q}^{(j)}\left(u \zeta^{\frac{1}{2}}\right) O\left(u^{-2 N-1}\right)$ as $u \rightarrow \infty$ uniformly for $0 \leq \tilde{q} \leq 2-\delta$, except near the zeros of the Bessel functions $\mathcal{C}_{q}^{(j)}\left(u \zeta^{\frac{1}{2}}\right)$.

The characterizing property of the two asymptotic solutions (3.16) and (3.17) is their recessiveness at $\zeta=0$ and $\zeta=\infty e^{\pi i}$, respectively. The corresponding recessive solutions of Jacobi's equation are $P_{n}^{(q, p)}\left(x e^{-\pi i}\right)$ and $Q_{n}^{(p, q)}(x)$, these being recessive at $x=-1(\arg (x)=\pi)$ and $x=\infty$, respectively. Now from the well-known behavior of Bessel functions [10, Chap. 12], we find that as $\zeta \rightarrow 0$,

$$
W_{2 N+1}^{(0)}(u, \tilde{q}, \zeta) \sim \frac{2 \zeta^{\frac{1}{2}(q+1)}}{\Gamma(q+1)}\left(\frac{u}{2}\right)^{q}\left[1+\sum_{s=1}^{N} \frac{\lambda_{s}(\tilde{q})}{u^{2 s}}+\tilde{q} \sum_{s=0}^{N-1} \frac{B_{s}(\tilde{q}, 0)}{u^{2 s+1}}\right] .
$$

Thus we have the relation

$$
P_{n}^{(q, p)}\left(x e^{-\pi i}\right)=\frac{D_{2 N+1}^{(0)}(u, \tilde{q})}{\zeta^{\frac{1}{2}}(1-x)^{\frac{1}{2} p+\frac{1}{4}}(x+1)^{\frac{1}{2} q}}\left(\frac{\zeta-\tilde{q}^{2}}{x-x_{t}(\tilde{q})}\right)^{\frac{1}{4}} W_{2 N+1}^{(0)}(u, \tilde{q}, \zeta),
$$

for some constant $D_{2 N+1}^{(0)}(u, \tilde{q})$. We determine this by letting $x \rightarrow-1$, and as a result deduce from (1.12) (with $p$ and $q$ interchanged and $x$ replaced by $-x),(3.12),(3.20)$, and (3.21) that

$$
\begin{aligned}
& D_{2 N+1}^{(0)}(u, \tilde{q})=\frac{2^{\frac{1}{2} p+\frac{3}{2} q-1} e^{q}(2 u-q)^{u-\frac{1}{2} q} \Gamma(n+q+1)}{(2 u+q)^{u+\frac{1}{2} q}} \Gamma(n+1) \\
& \times\left[1+\sum_{s=1}^{N} \frac{\lambda_{s}(\tilde{q})}{u^{2 s}}+\tilde{q} \sum_{s=0}^{N-1} \frac{B_{s}(\tilde{q}, 0)}{u^{2 s+1}}\right]^{-1} .
\end{aligned}
$$

Next we can identify the two solutions of (3.5) which are recessive at infinity, and this results in the relationship

$$
Q_{n}^{(p, q)}(x)=\frac{D_{2 N+1}^{(1)}(u, \tilde{q})}{\zeta^{\frac{1}{2}}(1-x)^{\frac{1}{2} p+\frac{1}{4}}(x+1)^{\frac{1}{2} q}}\left(\frac{\zeta-\tilde{q}^{2}}{x-x_{t}(\tilde{q})}\right)^{\frac{1}{4}} W_{2 N+1}^{(1)}(u, \tilde{q}, \zeta)
$$


where again $D_{2 N+1}^{(1)}(u, \tilde{q})$ is some constant which is to be determined. One method of finding this constant is to allow $x \rightarrow-1+i 0(\arg (x)=\pi)$ on both sides of (3.23), assuming temporarily that $q>0$. Using the well-known identity $H_{q}^{(1)}(z)=$ $J_{q}(z)+i Y_{q}(z)$ in (3.17) with [10, Chap. 12, Eqs. (1.07) and (1.08)], we find for the right-hand side of (3.23) that

$$
\begin{aligned}
W_{2 N+1}^{(1)}(u, \tilde{q}, \zeta) \sim-\frac{i \Gamma(q)}{\pi} & \left(\frac{2}{u}\right)^{q} \zeta^{\frac{1}{2}(1-q)} \\
& \times\left[1+\sum_{s=1}^{N} \frac{\lambda_{s}(\tilde{q})}{u^{2 s}}-\tilde{q} \sum_{s=0}^{N-1} \frac{B_{s}(\tilde{q}, 0)}{u^{2 s+1}}+\delta_{2 N+1}^{(1)}(u, \tilde{q})\right]
\end{aligned}
$$

as $\zeta \rightarrow 0$ with $q>0$ where

$$
\delta_{2 N+1}^{(1)}(u, \tilde{q})=\lim _{\zeta \rightarrow 0}\left\{\zeta^{\frac{1}{2}} H_{q}^{(1)}\left(u \zeta^{\frac{1}{2}}\right)\right\}^{-1} \varepsilon_{2 N+1}^{(1)}(u, \tilde{q}, \zeta) .
$$

Then from (1.19), (3.12), (3.23), and (3.24), we deduce that

$$
\begin{aligned}
& D_{2 N+1}^{(1)}(u, \tilde{q})=-\pi i e^{-(n+p) \pi i} \frac{2^{\frac{1}{2}(p-q)-1} \Gamma(n+p+1)}{e^{q} \Gamma(n+p+q+1)}(2 u+q)^{u+\frac{1}{2} q}(2 u-q)^{-u+\frac{1}{2} q} \\
& \times\left[1+\sum_{s=1}^{N} \frac{\lambda_{s}(\tilde{q})}{u^{2 s}}-\tilde{q} \sum_{s=0}^{N-1} \frac{B_{s}(\tilde{q}, 0)}{u^{2 s+1}}+\delta_{2 N+1}^{(1)}(u, \tilde{q})\right]^{-1}
\end{aligned}
$$

By analytic continuation this representation holds for $q=0$ as well. The constant $\delta_{2 N+1}^{(1)}(u, \tilde{q})$, whose existence is guaranteed by the existence of the coefficient $D_{2 N+1}^{(1)}(u, \tilde{q})$, can be bounded via [4, Eq. (5.16)] and is $O\left(u^{-2 N-1}\right)$ as $u \rightarrow \infty$ uniformly for $0 \leq \tilde{q} \leq 2-\delta$.

We now show how both $D_{2 N+1}^{(0)}(u, \tilde{q})$ and $D_{2 N+1}^{(1)}(u, \tilde{q})$ can be computed more easily and, as a by-product, give a scheme for easily calculating the constants $\left\{\lambda_{s}(\tilde{q})\right\}_{s=1}^{N}$ and $\left\{B_{s}(\tilde{q}, 0)\right\}_{s=0}^{N-1}$. Firstly, consider a circuit in the positive sense around the singularity $x=-1$. In the $\zeta$-plane this corresponds to a circuit in the positive sense around $\zeta=0$, and hence $\zeta^{\frac{1}{2}} \rightarrow \zeta^{\frac{1}{2}} e^{\pi i}$ as $x \rightarrow-1+(x+1) e^{2 \pi i}$. Therefore, on inserting (3.21) and (3.23) into (1.27), and using $H_{q}^{(1)}(z)=J_{q}(z)+i Y_{q}(z)$ and the well-known continuation $H_{q}^{(1)}\left(z e^{\pi i}\right)=-e^{-q \pi i}\left\{J_{q}(z)-i Y_{q}(z)\right\}$, we find that all the $Y$ terms cancel, and as a result the following relationship between the constants $D_{2 N+1}^{(0)}(u, \tilde{q})$ and $D_{2 N+1}^{(1)}(u, \tilde{q})$

$$
\begin{aligned}
\pi i D_{2 N+1}^{(0)}(u, \tilde{q}) & \left\{\mathcal{J}_{2 N+1}(u, \tilde{q}, \zeta)+\varepsilon_{2 N+1}^{(0)}(u, \tilde{q}, \zeta)\right\}=-e^{(p+n) \pi i} D_{2 N+1}^{(1)}(u, \tilde{q}) \\
& \times\left\{\mathcal{J}_{2 N+1}(u, \tilde{q}, \zeta)+\varepsilon_{2 N+1}^{(1)}(u, \tilde{q}, \zeta)+e^{q \pi i} \varepsilon_{2 N+1}^{(1)}\left(u, \tilde{q}, \zeta e^{2 \pi i}\right)\right\}
\end{aligned}
$$

where

$$
\mathcal{J}_{2 N+1}(u, \tilde{q}, \zeta)=2 \zeta^{\frac{1}{2}} J_{q}\left(u \zeta^{\frac{1}{2}}\right) \sum_{s=0}^{N} \frac{A_{s}(\tilde{q}, \zeta)}{u^{2 s}}+\frac{2 \zeta}{u} J_{q}^{\prime}\left(u \zeta^{\frac{1}{2}}\right) \sum_{s=0}^{N-1} \frac{B_{s}(\tilde{q}, \zeta)}{u^{2 s}}
$$


Since the left-hand side of (3.27) represents a solution of (3.5) which is recessive at $\zeta=0$, it follows of course that the function on the right-hand side is the same recessive solution of (3.5) (to within a multiplicative factor). Hence, on dividing both sides by $\mathcal{J}_{2 N+1}(u, \tilde{q}, \zeta)$ and letting $\zeta \rightarrow 0$, we can assert that

$$
\pi i D_{2 N+1}^{(0)}(u, \tilde{q})=-e^{(n+p) \pi i}\left\{1+O\left(u^{-2 N-1}\right)\right\} D_{2 N+1}^{(1)}(u, \tilde{q}) .
$$

Then, on inserting (3.22) and (3.26) into (3.29), we find that

$$
\begin{gathered}
\left(1+\sum_{s=1}^{N} \frac{\lambda_{s}(\tilde{q})}{u^{2 s}}+\tilde{q} \sum_{s=0}^{N-1} \frac{B_{s}(\tilde{q}, 0)}{u^{2 s+1}}\right)\left(1+\sum_{s=1}^{N} \frac{\lambda_{s}(\tilde{q})}{u^{2 s}}-\tilde{q} \sum_{s=0}^{N-1} \frac{B_{s}(\tilde{q}, 0)}{u^{2 s+1}}+O\left(u^{-2 N-1}\right)\right)^{-1} \\
=\frac{2^{2 q} e^{2 q}(2 u-q)^{2 u-q} \Gamma(n+p+q+1) \Gamma(n+q+1)}{(2 u+q)^{2 u+q} \Gamma(n+p+1) \Gamma(n+1)}
\end{gathered}
$$

Recall that the integration constants $\left\{\lambda_{s}(\tilde{q})\right\}_{s=1}^{N}$ are at our disposal. Let us now choose these so that

$$
\begin{aligned}
\left(1+\sum_{s=1}^{N} \frac{\lambda_{s}(\tilde{q})}{u^{2 s}}+\tilde{q} \sum_{s=0}^{N-1} \frac{B_{s}(\tilde{q}, 0)}{u^{2 s+1}}\right)\left(1+\sum_{s=1}^{N} \frac{\lambda_{s}(\tilde{q})}{u^{2 s}}-\tilde{q} \sum_{s=0}^{N-1} \frac{B_{s}(\tilde{q}, 0)}{u^{2 s+1}}\right) \\
=1+O\left(u^{-2 N-1}\right) .
\end{aligned}
$$

This is achieved by selecting each constant $\lambda_{s}(\tilde{q})(s=1,2, \ldots, N)$ in turn so that the coefficient of $u^{-2 s}$ in the expanded series on the left-hand side of (3.31) vanishes: note that the coefficients of the odd powers $u^{-2 s-1}$ are zero regardless of the choice of $\left\{\lambda_{s}(\tilde{q})\right\}_{s=1}^{N}$. Then, on multiplying the corresponding sides of (3.30) and (3.31), and then taking square roots, we arrive at

$$
\begin{aligned}
1+\sum_{s=1}^{N} \frac{\lambda_{s}(\tilde{q})}{u^{2 s}}+\tilde{q} & \sum_{s=0}^{N-1} \frac{B_{s}(\tilde{q}, 0)}{u^{2 s+1}}=\frac{2^{q} e^{q}(2 u-q)^{u-\frac{1}{2} q}}{(2 u+q)^{u+\frac{1}{2} q}} \\
& \times\left[\frac{\Gamma(n+p+q+1) \Gamma(n+q+1)}{\Gamma(n+p+1) \Gamma(n+1)}\left\{1+O\left(u^{-2 N-1}\right)\right\}\right]^{\frac{1}{2}}
\end{aligned}
$$

and hence from $(3.30)$ or $(3.31)$

$$
\begin{aligned}
1+\sum_{s=1}^{N} \frac{\lambda_{s}(\tilde{q})}{u^{2 s}}-\tilde{q} \sum_{s=0}^{N-1} \frac{B_{s}(\tilde{q}, 0)}{u^{2 s+1}}+\delta_{2 N+1}^{(1)}(u, \tilde{q})=\frac{(2 u+q)^{u+\frac{1}{2} q}}{2^{q} e^{q}(2 u-q)^{u-\frac{1}{2} q}} \\
\times\left[\frac{\Gamma(n+p+1) \Gamma(n+1)}{\Gamma(n+p+q+1) \Gamma(n+q+1)}\left\{1+O\left(u^{-2 N-1}\right)\right\}\right]^{\frac{1}{2}} .
\end{aligned}
$$

From (3.22), (3.26), (3.32), and (3.33), we consequently arrive at the following representations

$$
D_{2 N+1}^{(0)}(u, \tilde{q})=2^{\frac{1}{2}(p+q)-1}\left[\frac{\Gamma(n+p+1) \Gamma(n+q+1)}{\Gamma(n+p+q+1) \Gamma(n+1)}\right]^{\frac{1}{2}}\left\{1+O\left(u^{-2 N-1}\right)\right\}
$$

and 


$$
\begin{aligned}
& D_{2 N+1}^{(1)}(u, \tilde{q})=-\pi i e^{-(n+p) \pi i} 2^{\frac{1}{2}(p+q)-1} \\
& \times\left[\frac{\Gamma(n+p+1) \Gamma(n+q+1)}{\Gamma(n+p+q+1) \Gamma(n+1)}\right]^{\frac{1}{2}}\left\{1+O\left(u^{-2 N-1}\right)\right\} .
\end{aligned}
$$

These are simple formulas for the coefficients, but they only provide an approximation since they involve a small but unknown constant. However, the coefficient $D_{2 N+1}^{(0)}(u, \tilde{q})$ can be computed to arbitrary accuracy by first computing the constants $\left\{\lambda_{s}(\tilde{q})\right\}_{s=1}^{N}$ and $\left\{B_{s}(\tilde{q}, 0)\right\}_{s=0}^{N-1}$ via the formal expansion (see (2.1) and (3.32))

$$
\begin{array}{r}
\left(\frac{2 e}{u}\right)^{u \tilde{q}}\left(4-\tilde{q}^{2}\right)^{-\frac{1}{2} u \tilde{q}}\left(\frac{2-\tilde{q}}{2+\tilde{q}}\right)^{u}\left[\frac{\Gamma\left(u\left(1-\frac{1}{2} \tilde{q}\right)+\frac{1}{2} p+\frac{1}{2}\right) \Gamma\left(u\left(1-\frac{1}{2} \tilde{q}\right)-\frac{1}{2} p+\frac{1}{2}\right)}{\Gamma\left(u\left(1+\frac{1}{2} \tilde{q}\right)+\frac{1}{2} p+\frac{1}{2}\right) \Gamma\left(u\left(1+\frac{1}{2} \tilde{q}\right)-\frac{1}{2} p+\frac{1}{2}\right)}\right]^{\frac{1}{2}} \\
\sim 1+\sum_{s=1}^{\infty} \frac{\lambda_{s}(\tilde{q})}{u^{2 s}}+\tilde{q} \sum_{s=0}^{\infty} \frac{B_{s}(\tilde{q}, 0)}{u^{2 s+1}} .
\end{array}
$$

Then, after computing the first $N$ such terms of each set, their insertion into (3.22) yields an exact representation for $D_{2 N+1}^{(0)}(u, \tilde{q})$ as required.

On using Stirling's formula for the asymptotic expansion of the Gamma function, and with the aid of a symbolic software package such as MAPLE, the constants $\left\{\lambda_{s}(\tilde{q})\right\}_{s=1}^{N}$ and $\left\{B_{s}(\tilde{q}, 0)\right\}_{s=0}^{N-1}$ can readily be computed via an expansion of the lefthand side of (3.36) in inverse powers of $u$. For instance, we find the first two of each set to be

$$
\begin{gathered}
B_{0}(\tilde{q}, 0)=\frac{1-3 p^{2}}{6\left(4-\tilde{q}^{2}\right)} \\
\lambda_{1}(\tilde{q})=\frac{\tilde{q}^{2}\left(1-3 p^{2}\right)^{2}}{72\left(4-\tilde{q}^{2}\right)^{2}} \\
B_{1}(\tilde{q}, 0)=\frac{5 \tilde{q}^{2}\left(1-3 p^{2}\right)^{3}-36\left(\tilde{q}^{2}+12\right)\left(15 p^{4}-30 p^{2}+7\right)}{6480\left(4-\tilde{q}^{2}\right)^{3}}
\end{gathered}
$$

and

$$
\lambda_{2}(\tilde{q})=\frac{\tilde{q}^{2}\left(1-3 p^{2}\right)\left[5 \tilde{q}^{2}\left(1-3 p^{2}\right)^{3}-144\left(\tilde{q}^{2}+12\right)\left(15 p^{4}-30 p^{2}+7\right)\right]}{155520\left(4-\tilde{q}^{2}\right)^{4}}
$$

Turning to the coefficient $D_{2 N+1}^{(1)}(u, \tilde{q})$, we now have two representations, namely (3.26) and (3.35), but neither provide a means of calculating this coefficient to arbitrary accuracy. However, an alternative method of determining $D_{2 N+1}^{(1)}(u, \tilde{q})$ is to allow $\zeta \rightarrow-\infty(\arg (\zeta)=\pi)$ in the relation (3.23). To this end we see from [10, Chap. 7, Eq. (4.03)] and (3.17) that as $\zeta \rightarrow-\infty$

$$
\begin{aligned}
W_{2 N+1}^{(1)}(u, \tilde{q}, \zeta) \sim\left(\frac{2}{\pi u}\right)^{\frac{1}{2}} \zeta^{\frac{1}{4}} \exp \{i( & \left.\left.u \zeta^{\frac{1}{2}}-\frac{1}{2} q \pi-\frac{1}{4} \pi\right)\right\} \\
& \times\left[1+\sum_{s=1}^{N} \frac{A_{s}(\tilde{q}, \infty)}{u^{2 s}}+\sum_{s=0}^{N-1} \frac{b_{s}(\tilde{q}, \infty)}{u^{2 s+1}}\right]
\end{aligned}
$$

where

$$
A_{s}(\tilde{q}, \infty)=\lim _{\zeta \rightarrow-\infty} A_{s}(\tilde{q}, \zeta), \quad b_{s}(\tilde{q}, \infty)=i \lim _{\zeta \rightarrow-\infty} \zeta^{\frac{1}{2}} B_{s}(\tilde{q}, \zeta)
$$


Hence we find, from (1.21), (3.15), (3.23), and (3.41), that

$$
\begin{aligned}
D_{2 N+1}^{(1)}(u, \tilde{q})=-i e^{-(n+p) \pi i} K_{n}^{(p, q)} & \sqrt{\frac{u \pi}{2}} 2^{5 u-n}(2-\tilde{q})^{\frac{1}{2} q-u}(2+\tilde{q})^{-\frac{1}{2} q-u} \\
\times & {\left[1+\sum_{s=1}^{N} \frac{A_{s}(\tilde{q}, \infty)}{u^{2 s}}+\sum_{s=0}^{N-1} \frac{b_{s}(\tilde{q}, \infty)}{u^{2 s+1}}\right]^{-1} }
\end{aligned}
$$

This gives the exact representation for $D_{2 N+1}^{(1)}(u, \tilde{q})$ that we were seeking. Rather than calculating the limits (3.42) directly (which can be difficult), we instead use (3.29), (3.34), and (3.43). From these we arrive at the relation

$$
\begin{aligned}
& \sqrt{\frac{u}{\pi}}\left(\frac{2-\tilde{q}}{2+\tilde{q}}\right)^{\frac{1}{2} u \tilde{q}}\left(\frac{16}{4-\tilde{q}^{2}}\right)^{u} \frac{\left[\Gamma\left(u\left(1+\frac{1}{2} \tilde{q}\right)+\frac{1}{2} p+\frac{1}{2}\right) \Gamma\left(u\left(1+\frac{1}{2} \tilde{q}\right)-\frac{1}{2} p+\frac{1}{2}\right)\right]^{\frac{1}{2}}}{\Gamma(2 u+1)} \\
\times & {\left[\Gamma\left(u\left(1-\frac{1}{2} \tilde{q}\right)+\frac{1}{2} p+\frac{1}{2}\right) \Gamma\left(u\left(1-\frac{1}{2} \tilde{q}\right)-\frac{1}{2} p+\frac{1}{2}\right)\right]^{\frac{1}{2}} \sim 1+\sum_{s=1}^{\infty} \frac{A_{s}(\tilde{q}, \infty)}{u^{2 s}}+\sum_{s=0}^{\infty} \frac{b_{s}(\tilde{q}, \infty)}{u^{2 s+1}} }
\end{aligned}
$$

and by expanding the left-hand side in a similar manner to (3.36), we can compute $\left\{A_{s}(\tilde{q}, \infty)\right\}_{s=1}^{N}$ and $\left\{b_{s}(\tilde{q}, \infty)\right\}_{s=1}^{N-1}$ quite readily. For instance, we find that the first four terms are

$$
\begin{gathered}
b_{0}(\tilde{q}, \infty)=\frac{24 p^{2}+\tilde{q}^{2}-12}{24\left(4-\tilde{q}^{2}\right)}, \\
A_{1}(\tilde{q}, \infty)=\frac{\left(24 p^{2}+\tilde{q}^{2}-12\right)^{2}}{1152\left(4-\tilde{q}^{2}\right)^{2}}, \\
b_{1}(\tilde{q}, \infty)=\frac{\left(2 p^{2}-3\right)\left(4 p^{4}+16 p^{2}-5\right)+p^{2} \tilde{q}^{2}\left(25 p^{2}-49\right)}{48\left(4-\tilde{q}^{2}\right)^{3}} \\
+\frac{\tilde{q}^{2}\left(360 p^{2} \tilde{q}^{2}-139 \tilde{q}^{4}+1548 \tilde{q}^{2}+92016\right)}{414720\left(4-\tilde{q}^{2}\right)^{3}}
\end{gathered}
$$

and

$$
\begin{aligned}
& A_{2}(\tilde{q}, \infty)=\left(4-\tilde{q}^{2}\right)^{-4}\left(24 p^{2}+\tilde{q}^{2}-12\right)\left[\frac{p^{2}\left(4 p^{2}+58 p^{2}-125\right)}{2304}\right. \\
& \left.\quad+\frac{p^{2} \tilde{q}^{2}\left(2328 p^{2}+\tilde{q}^{2}-4632\right)}{110592}-\frac{571 \tilde{q}^{6}-6732 \tilde{q}^{4}-361584 \tilde{q}^{2}-544320}{39813120}\right] .
\end{aligned}
$$

Finally, to obtain an asymptotic expansion for the Jacobi function $P_{n}^{(p, q)}(x)$, we insert (3.21) and (3.23) into the right-hand side of (1.23), and invoke (3.29), and as a result, we obtain the relation

$$
\begin{aligned}
& P_{n}^{(p, q)}(x)=\frac{D_{2 N+1}^{(0)}(u, \tilde{q})}{\zeta^{\frac{1}{2}}(1-x)^{\frac{1}{2} p+\frac{1}{4}}(x+1)^{\frac{1}{2} q}}\left(\frac{\zeta-\tilde{q}^{2}}{x-x_{t}(\tilde{q})}\right)^{\frac{1}{4}}\left[2 \zeta^{\frac{1}{2}} \mathcal{C}_{q}\left(u \zeta^{\frac{1}{2}}\right) \sum_{s=0}^{N} \frac{A_{s}(\tilde{q}, \zeta)}{u^{2 s}}\right. \\
& \left.+\frac{2 \zeta}{u} \mathcal{C}_{q}^{\prime}\left(u \zeta^{\frac{1}{2}}\right) \sum_{s=0}^{N-1} \frac{B_{s}(\tilde{q}, \zeta)}{u^{2 s}}+e^{n \pi i} \varepsilon_{2 N+1}^{(0)}(u, \tilde{q}, \zeta)-2 i \sin (n \pi) \varepsilon_{2 N+1}^{(1)}(u, \tilde{q}, \zeta)\right]
\end{aligned}
$$


where

$$
\mathcal{C}_{q}\left(u \zeta^{\frac{1}{2}}\right)=\cos (n \pi) J_{q}\left(u \zeta^{\frac{1}{2}}\right)+\sin (n \pi) Y_{q}\left(u \zeta^{\frac{1}{2}}\right) .
$$

This expansion is valid for the same values of the variable and parameters as the expansions (3.21) and (3.23), i.e., for all $x$ in the cut plane $0 \leq \arg (x+1) \leq 2 \pi$ except points at or near the singularity $x=1$, uniformly for large $n$, fixed $p$, and all $q$ satisfying (3.1).

Recall that $P_{n}^{(p, q)}(x)$ is the Jacobi polynomial, and hence is recessive at $x=-1$, when $n \in \mathbf{N}$, and indeed in this case the right-hand side of (3.49) reduces to $(-1)^{n}$ times the right-hand side of (3.21), (as expected from (1.11)). This confirms that (3.49) holds for all non-negative values of $n$ (integer and non-integer).

4. $p$ and $q$ large and equal, and $n$ small or large: the ultraspherical polynomials. In this and the following section, we consider the case where both $p$ and $q$ are large. We first rewrite (2.3) in the form

$$
\frac{d^{2} w}{d x^{2}}=\left\{u^{2} \frac{\left(x-x_{1}\right)\left(x-x_{2}\right)}{\left(x^{2}-1\right)^{2}}-\frac{3+x^{2}}{4\left(x^{2}-1\right)^{2}}\right\} w
$$

where

$$
x_{1,2}=\frac{1}{4}\left(\tilde{q}^{2}-\tilde{p}^{2}\right) \mp \frac{1}{4} \sqrt{\left\{4-(\tilde{p}+\tilde{q})^{2}\right\}\left\{4-(\tilde{p}-\tilde{q})^{2}\right\}}
$$

and therefore we perceive that there now are two turning points. We shall focus on the case where $p=q$, which when $n \in \mathrm{N}$ leads to the ultraspherical polynomials as solutions of Jacobi's equation (1.1) (see (1.5)). As in the previous two sections, we do not insist that $n \in \mathbf{N}$, and we also provide asymptotic approximations for companion (non-polynomial) solutions.

When $p=q,(4.1)$ becomes .

$$
\frac{d^{2} w}{d x^{2}}=\left\{u^{2} \frac{x^{2}-\beta}{\left(x^{2}-1\right)^{2}}-\frac{3+x^{2}}{4\left(x^{2}-1\right)^{2}}\right\} w
$$

where

$$
\beta=1-\tilde{p}^{2}
$$

and now from (1.2)

$$
u=n+p+\frac{1}{2} \rightarrow \infty
$$

Note that our general assumptions $0 \leq p<\infty$ and $0 \leq n<\infty$ now imply that $0 \leq \tilde{p}<1$, where $\tilde{p}$ is defined by (2.1). Hence $0<\beta \leq 1$.

The differential equation (4.3) is characterized by having two turning points at $x= \pm \sqrt{\beta}$, which coalesce with one another when $\beta \rightarrow 0(\tilde{p} \rightarrow 1)$, and coalesce with the poles at $x= \pm 1$ when $\beta \rightarrow 1(\tilde{p} \rightarrow 0)$. In this section, we allow the turning points to coalesce with one another, but not with the poles. Thus we shall assume that $\delta \leq \tilde{p}<1$ or equivalently $p \rightarrow \infty$, and for all $n$ satisfying

$$
0 \leq n \leq A p
$$


where $A$ is an arbitrary positive constant (which is independent of $p$ ).

Although a general theory of coalescing turning points exists [11], it only holds for real values of the independent variable. However, since (4.3) is an even equation in $x$, we can make the following simple preliminary Liouville transformation

$$
t=x^{2}, \quad y(t)=t^{\frac{1}{4}} w(x),
$$

to recast it in the form

$$
\frac{d^{2} y}{d t^{2}}=\left\{u^{2} \frac{t-\beta}{4 t(t-1)^{2}}-\frac{4 t^{2}-3 t+3}{16 t^{2}(t-1)^{2}}\right\} y .
$$

In what follows, we assume that $0 \leq \arg (x) \leq \pi$, and hence, $0 \leq \arg (t) \leq 2 \pi$. Extension of the following asymptotic results to the range $-\pi<\arg (x) \leq 0$ can be achieved via the analytic continuation formulas

$$
P_{n}^{(p, p)}\left(x e^{-\pi i}\right)=e^{-n \pi i} P_{n}^{(p, p)}(x)-\frac{2 e^{p \pi i} \sin (n \pi)}{\pi} Q_{n}^{(p, p)}(x)
$$

and

$$
Q_{n}^{(p, p)}\left(x e^{-\pi i}\right)=-e^{(n+2 p) \pi i} Q_{n}^{(p, p)}(x) .
$$

It is now clear that we again can apply the theory of a coalescing turning point and simple pole of [6] to (4.8). To this end, let us now make a second Liouville transformation

$$
\begin{gathered}
\left(\frac{d \eta}{d t}\right)^{2}=\frac{\eta(t-\beta)}{4 t(t-1)^{2}\left(\eta-\alpha^{*}\right)} \\
U(\eta)=\left(\frac{d \eta}{d t}\right)^{\frac{1}{2}} y(t)
\end{gathered}
$$

to transform (4.8) into the desired form

$$
\frac{d^{2} U}{d \eta^{2}}=\left\{u^{2} \frac{\eta-\alpha^{*}}{\eta}+\frac{m^{2}-\frac{1}{4}}{\eta^{2}}+\frac{\psi^{*}\left(\alpha^{*}, \eta\right)}{\eta}\right\} U
$$

where, in the notation of $[6, \S 4], \alpha$ has been replaced by $\alpha^{*}, \psi$ by $\psi^{*}, W$ by $U$, and $\xi$ by $\eta$, (to avoid confusion with $\S 2$ above). Thus from [6, Eq. (4.6)]

$$
\alpha^{*}=\frac{1}{\pi} \int_{0}^{\beta}\left\{\frac{\beta-s}{s}\right\}^{1 / 2} \frac{d s}{1-s}=1-\sqrt{1-\beta}=1-\tilde{p} .
$$

It turns out that, with the choice $m=\frac{1}{4}$, the function $\psi^{*}\left(\alpha^{*}, \eta\right)$ is given by

$$
\psi^{*}\left(\alpha^{*}, \eta\right)=\frac{3 \eta+2 \alpha^{*}}{16\left(\eta-\alpha^{*}\right)^{2}}+\frac{\left(\eta-\alpha^{*}\right)(1-t)\left(t-4 \tilde{p}^{2} t+\tilde{p}^{4}-1\right)}{4(t-\beta)^{3}}
$$

and is analytic at $\eta=0$ (see (4.27) below).

Therefore, the transformed equation takes the form

$$
\frac{d^{2} U}{d \eta^{2}}=\left\{u^{2} \frac{\eta-\alpha^{*}}{\eta}-\frac{3}{16 \eta^{2}}+\frac{\psi^{*}\left(\alpha^{*}, \eta\right)}{\eta}\right\} U
$$




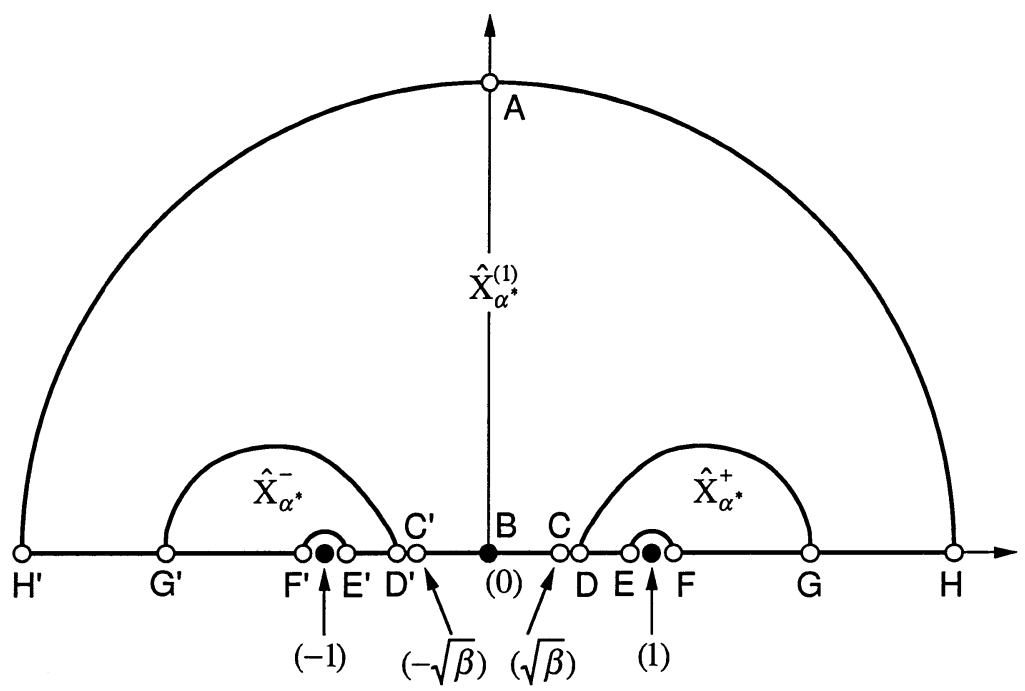

Figure 3(a): $x$ plane

and the corresponding Liouville transformation is explicitly given by

$$
U(\eta)=\frac{1}{(1-t)^{\frac{1}{2}}}\left\{\frac{\eta(t-\beta)}{t\left(\alpha^{*}-\eta\right)}\right\}^{\frac{1}{4}} y(t),
$$

with

$$
\int_{\alpha^{*}}^{\eta}\left\{\frac{\tau-\alpha^{*}}{\tau}\right\}^{\frac{1}{2}} d \tau=\frac{1}{2} \int_{\beta}^{t}\left\{\frac{s-\beta}{s}\right\}^{\frac{1}{2}} \frac{d s}{1-s} .
$$

Explicit integration of (4.18) yields the relationship (for complex values of the variables)

$$
\begin{aligned}
& \eta^{\frac{1}{2}}(\eta\left.-\alpha^{*}\right)^{\frac{1}{2}}-\frac{\alpha^{*}}{2} \ln \left\{\frac{2 \eta-\alpha^{*}+2 \eta^{\frac{1}{2}}\left(\eta-\alpha^{*}\right)^{\frac{1}{2}}}{\alpha^{*}}\right\}=\frac{\sqrt{1-\beta}}{2} \ln \left\{\frac{t}{\beta(1-t)}\right\} \\
&+\sqrt{1-\beta} \ln \left\{\left(\frac{t-\beta}{t}\right)^{\frac{1}{2}}+\sqrt{1-\beta}\right\}+\frac{1}{2} \ln \left(\frac{t}{\beta}\right)+\ln \left\{1-\left(\frac{t-\beta}{t}\right)^{\frac{1}{2}}\right\}
\end{aligned}
$$

As mentioned above, we choose $\arg (\eta)$ to lie in the interval $[0,2 \pi]$. The branches in (4.19) are chosen so that $\eta(t)$ is real and negative $(\arg (\eta)=\pi)$ when $t$ is negative $(\arg (t)=\pi)$, with $\eta(t)$ then being a continuous function of $t$ elsewhere in the cut plane $0 \leq \arg (t) \leq 2 \pi$. In particular $\eta(t)$ lies above or below the cut along the positive real axis when $t$ lies, respectively, above or below the cut from 0 to 1 .

The map of the upper half $x$ plane to the $t$ plane is depicted in Figures 3(a) and 3(b), and the corresponding domain in the $\eta$ plane is depicted in Figure 3(c). In Figure 3(c), the curve $F H$ in the $\eta$ plane corresponds to the upper lip of the cut from $t=1$ to $t=\infty$ and is given parametrically by

$$
\int_{\alpha^{*}}^{\eta}\left\{\frac{\tau-\alpha^{*}}{\tau}\right\}^{\frac{1}{2}} d \tau=s+\frac{1}{2}\left(1-\alpha^{*}\right) \pi i, \quad-\infty<s<\infty .
$$




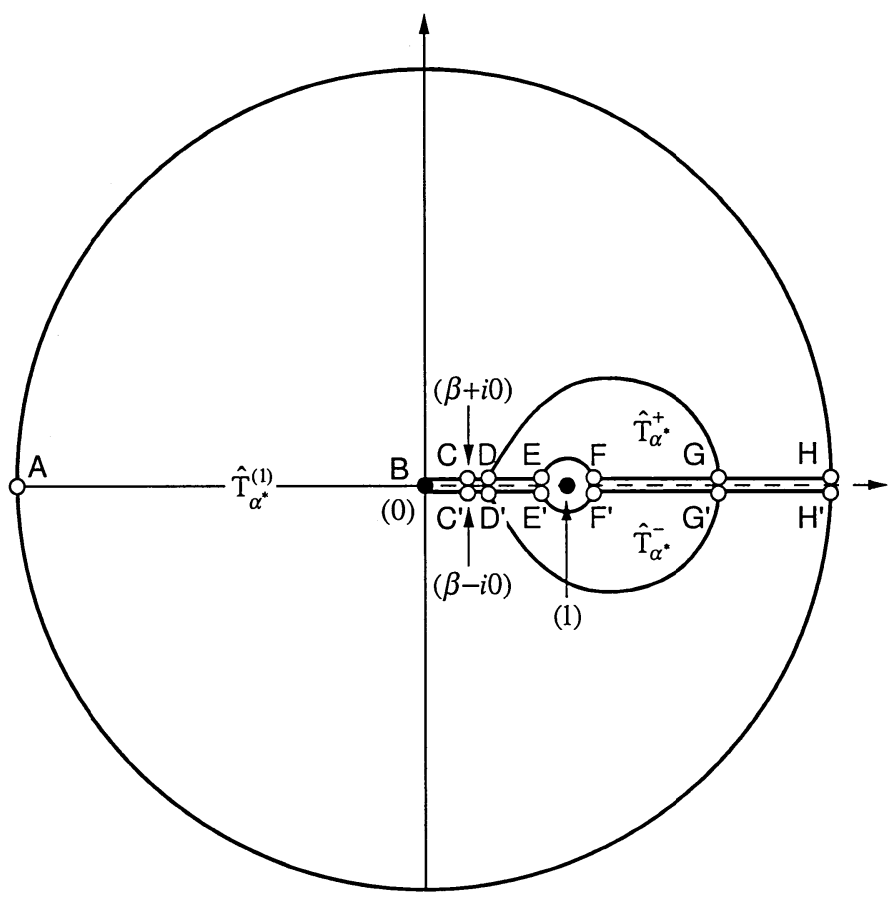

Figure $3(\mathrm{~b}): t$ plane

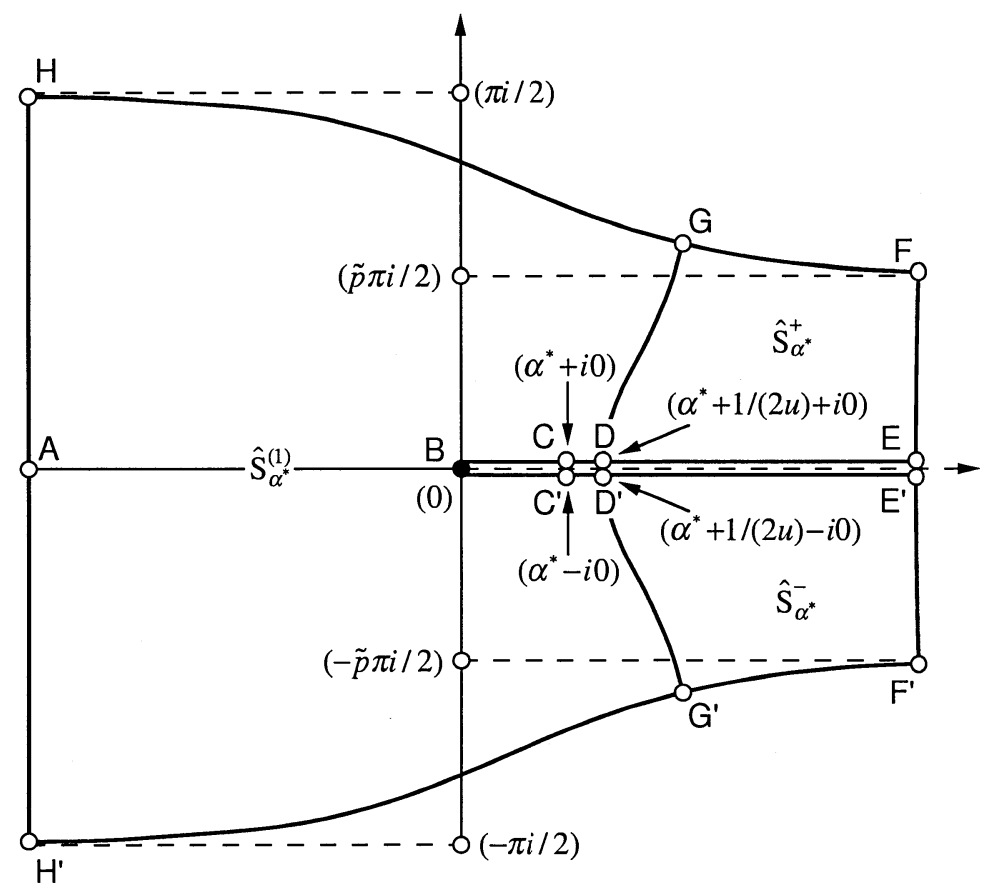

Figure 3(c): $\eta$ plane 
This curve has horizontal asymptotes of $\operatorname{Im} \eta=\frac{1}{2} \pi i$ and $\operatorname{Im} \eta=\frac{1}{2}\left(1-\alpha^{*}\right) \pi i=\frac{1}{2} \tilde{p} \pi i$ at $\operatorname{Re} \eta=-\infty$ and $\operatorname{Re} \eta=\infty$, respectively. The curve $F^{\prime} H^{\prime}$, corresponding to the lower lip of the cut from $t=1$ to $t=\infty$ is the conjugate of $F H$.

Also in Figure 3(c) three subdomains, $\hat{S}_{\alpha^{*}}^{(1)}, \hat{S}_{\alpha^{*}}^{+}, \hat{S}_{\alpha^{*}}^{-}$of the $\eta$ plane, are depicted. The domain $\hat{S}_{\alpha^{*}}^{(1)}$ is an extension of that given by [6, §4] to $0 \leq \arg (\eta)<2 \pi$ (where $\alpha$ has been replaced by $\left.\alpha^{*}\right)$. The cut $[0, \infty)$ separates the domain $\hat{S}_{\alpha^{*}}^{(3)}$ described in $[6, \S 4]$ into two components, which we have labelled $\hat{S}_{\alpha^{*}}^{+}$and $\hat{S}_{\alpha^{*}}^{-}$, and we note that $0 \leq \arg (\eta)<\frac{1}{2} \pi$ for $\eta \in \hat{S}_{\alpha^{*}}^{+}$and $\frac{3}{2} \pi<\arg (\eta) \leq 2 \pi$ for $\eta \in S_{\alpha^{*}}^{-}$. The curves $D G$ and $D^{\prime} G^{\prime}$ depicted in Figure 3(c), which along with the interval $\left(-\infty, \alpha^{*}+1 /(2 u)\right]$ separate the three domains, are given, respectively, by (cf. (2.25))

$$
\operatorname{Re} \int_{\alpha^{*}+1 /(2 u) \pm i 0}^{\eta}\left(\frac{t-\alpha^{*}}{t}\right)^{\frac{1}{2}} d t=0 .
$$

The $x$ domains corresponding to $\hat{S}_{\alpha^{*}}^{(1)}, \hat{S}_{\alpha^{*}}^{+}$, and $\hat{S}_{\alpha^{*}}^{-}$are labelled as $\hat{X}_{\alpha^{*}}^{(1)}, \hat{X}_{\alpha^{*}}^{+}$, and $\hat{X}_{\alpha^{*}}^{-}$, respectively, in Figure 3(a), and the corresponding $t$ domains are labelled as $\hat{T}_{\alpha^{*}}^{(1)}, \hat{T}_{\alpha^{*}}^{+}$, and $\hat{T}_{\alpha^{*}}^{-}$, respectively, in Figure $3(\mathrm{~b})$.

Next, from (4.19), we infer that $\operatorname{Re} \eta \rightarrow-\infty$ as $t \rightarrow \infty$ such that

$$
t \sim e^{\tilde{p} \pi i}(1+\tilde{p})\left(\frac{1+\tilde{p}}{4 e}\right)^{\tilde{p}} \eta^{1-\tilde{p}} e^{-2 \eta+1},
$$

and $\eta \rightarrow \infty \pm i 0$ as $t \rightarrow 1 \pm i 0(x \rightarrow \pm 1)$ such that

$$
t \sim 1-\frac{\tilde{p}^{2}}{(1+\tilde{p})}\left(\frac{4 e}{1+\tilde{p}}\right)^{\frac{1}{\tilde{p}}} \eta^{\frac{1}{\bar{p}}-1} \exp \left\{-\frac{2 \eta}{\tilde{p}}-1\right\} .
$$

In the special case when $\eta$ and $t$ are real with $0<\eta \leq \alpha^{*}(0<t \leq \beta)$ the relationship between the variables can be expressed as

$$
\int_{0}^{\eta}\left\{\frac{\alpha^{*}-\tau}{\tau}\right\}^{\frac{1}{2}} d \tau=\frac{1}{2} \int_{0}^{t}\left\{\frac{\beta-s}{s}\right\}^{\frac{1}{2}} \frac{d s}{1-s}
$$

which on integration yields

$$
\begin{aligned}
\eta^{\frac{1}{2}}\left(\alpha^{*}-\eta\right)^{\frac{1}{2}}+\frac{\alpha^{*}}{2} & \arccos \left(\frac{\alpha^{*}-2 \eta}{\alpha^{*}}\right) \\
& =\arccos \left\{\left(\frac{\beta-t}{\beta}\right)^{\frac{1}{2}}\right\}-\sqrt{1-\beta} \arccos \left\{\left(\frac{\beta-t}{\beta(1-t)}\right)^{\frac{1}{2}}\right\}
\end{aligned}
$$

The branches of the inverse cosines are such that they are continuous in $0<\eta \leq \alpha^{*}$ $(0<t \leq \beta)$, with the one on the left-hand side increasing montonically from 0 to $\pi$ when $\eta$ increases from 0 to $\alpha^{*}$, and the ones on the right-hand side increasing montonically from 0 to $\frac{1}{2} \pi$ as $t$ increases from 0 to $\beta$.

From (4.25), we find as $t \rightarrow 0$ that

$$
t=\frac{4}{1+\tilde{p}} \eta-\frac{4(5+7 \tilde{p})}{3(1+\tilde{p})^{3}} \eta^{2}+O\left(\eta^{3}\right)
$$


and hence from (4.15)

$$
\psi^{*}\left(\alpha^{*}, \eta\right)=-\frac{\alpha^{*}}{8\left(2-\alpha^{*}\right)^{2}}+O(\eta)
$$

We again can apply Theorem 2 of [6], with the above changes in notation of the variables. The identification of the Jacobi functions then follows in a similar manner to $\S 2$. Firstly, for the solutions which are recessive at $x=1(\eta=\infty+i 0)$, we find that for some constant $D_{4}\left(u, \alpha^{*}\right)$

$$
P_{n}^{(p, p)}(x)=D_{4}\left(u, \alpha^{*}\right)\left(1-x^{2}\right)^{-\frac{1}{2} p}\left\{\frac{\eta-\alpha^{*}}{\eta\left(x^{2}-\beta\right)}\right\}^{\frac{1}{4}}\left\{\mathcal{U}_{\frac{1}{2} u \alpha^{*}, \frac{1}{4}}^{(4)}(2 u \eta)+\hat{\varepsilon}_{4}^{+}\left(u, \alpha^{*}, \eta\right)\right\}
$$

The asymptotic solution on the right-hand side of this equation is $\hat{W}^{(4)}$, as given by Theorem 2 of [6], where the so-called reference point is chosen at $\eta=\infty+i 0$. The $\eta$ region of validity of this approximation is $\hat{S}_{\alpha^{*}}^{+}$, i.e., the domain in which the error term, which we have denoted by $\hat{\varepsilon}_{4}^{+}\left(u, \alpha^{*}, \eta\right)$, satisfies the bound [6, Eq. (4.80)] for $j=4$.

By comparing both sides of $(4.28)$ at $x=1(\eta=\infty+i 0)$, we find, on employing (4.23), that

$$
D_{4}\left(u, \alpha^{*}\right)=-i e^{-\frac{1}{2} n \pi i} \frac{\Gamma(n+p+1) 2^{p} p^{p+\frac{1}{2}}(u+p)^{-\frac{1}{2}(u+p)}(u-p)^{\frac{1}{2}(u-p)}}{\sqrt{u} \Gamma(n+1) \Gamma(p+1)} .
$$

In a similar manner, we find that for the solutions which are recessive at $x=-1$ $(\eta=\infty-i 0)$

$$
P_{n}^{(p, p)}\left(x e^{-\pi i}\right)=D_{4}\left(u, \alpha^{*}\right)\left(1-x^{2}\right)^{-\frac{1}{2} p}\left\{\frac{\eta-\alpha^{*}}{\eta\left(x^{2}-\beta\right)}\right\}^{\frac{1}{4}}\left\{\hat{\mathcal{U}}_{\frac{1}{2} u \alpha^{*}, \frac{1}{4}}^{(4)}(2 u \eta)+\hat{\varepsilon}_{4}^{-}\left(u, \alpha^{*}, \eta\right)\right\}
$$

where the asymptotic solution on the right-hand side is again $\hat{W}^{(4)}$, as given by Theorem 2 of [6], but this time where the reference point is chosen at $\eta=\infty-i 0$ (with the corresponding error term denoted as $\hat{\epsilon}_{4}^{-}\left(u, \alpha^{*}, \eta\right)$ to distinguish it from the error term $\hat{\varepsilon}_{4}^{+}\left(u, \alpha^{*}, \eta\right)$ given in(4.28) above). The $\eta$ region of validity of this approximation is $\hat{S}_{\alpha^{*}}^{-}$. Note that even though the right-hand sides of (4.28) and (4.30) are seemingly identical, the error terms differ when $n \notin \mathrm{N}$, and as such the two asymptotic solutions are linearly independent in this case, with the domains of asymptotic validity being disjoint.

Our third identification, of recessive solutions at infinity, yields

$$
Q_{n}^{(p, p)}(x)=D_{1}\left(u, \alpha^{*}\right)\left(x^{2}-1\right)^{-\frac{1}{2} p}\left\{\frac{\eta-\alpha^{*}}{\eta\left(x^{2}-\beta\right)}\right\}^{\frac{1}{4}}\left\{\hat{\mathcal{U}}_{\frac{1}{2} u \alpha^{*}, \frac{1}{4}}^{(1)}(2 u \eta)+\hat{\varepsilon}_{1}\left(u, \alpha^{*}, \eta\right)\right\}
$$

where, from a comparison at $x=\infty(\operatorname{Re} \eta=-\infty)$ and use of (4.22), we have

$$
D_{1}\left(u, \alpha^{*}\right)=e^{-\frac{1}{2}(n+p) \pi i} \frac{K_{n}^{(p, q)} 2^{2 u+p+\frac{1}{2}} u^{u}}{(u+p)^{\frac{1}{2}(u+p)}(u-p)^{\frac{1}{2}(u-p)}} .
$$


This approximation is valid for all $\eta$ and $x$ under consideration.

It remains to obtain an approximation for $P_{n}^{(p, p)}(x)$ which is valid for points excluded from the region of validity of (4.28), i.e., for $\eta \in \hat{S}_{\alpha^{*}}^{(1)} \cup \hat{S}_{\alpha^{*}}^{-}$. We shall do this by identifying the asymptotic solution $\hat{W}^{(0)}$ of Theorem 2 of [6] which is recessive at $\eta=0$ with the Jacobi functions. With regard to the original equation (4.3), the point $\eta=0$ corresponds to $x=0$, which actually is just an ordinary point of the equation. The appearance of $t=0$ and, correspondingly, $\eta=0$, as a pole in the differential equations (4.8) and (4.13), respectively, comes from the fact that the preliminary transformation (4.7) is not regular at that point. Thus in order to match $\hat{W}^{(0)}$, we seek a solution of (4.3) which vanishes at $x=0$, i.e., one which is $O(x)$ as $x \rightarrow 0$.

To this end we first utilize (1.6), (1.9), and [1, Eqs. 15.1.24 and 15.2.1], and from these it can be shown that

$$
\begin{aligned}
P_{n}^{(p, p)}(x)=\frac{\cos \left(\frac{1}{2} n \pi\right) \Gamma(n+p+1)}{\sqrt{\pi} \Gamma\left(\frac{1}{2} n+\frac{1}{2}\right)} & \\
+ & \frac{\sin \left(\frac{1}{2} n \pi\right) \Gamma(n+p+1) \Gamma\left(\frac{1}{2} n\right)}{\sqrt{\pi} \Gamma(n) \Gamma\left(\frac{1}{2} n+p+\frac{1}{2}\right)} x+O\left(x^{2}\right)
\end{aligned}
$$

as $x \rightarrow 0$, and

$$
\begin{gathered}
Q_{n}^{(p, p)}(x)=-\frac{i e^{-\left(p+\frac{1}{2} n\right) \pi i} \sqrt{\pi} \Gamma(n+p+1) \Gamma\left(\frac{1}{2} n+\frac{1}{2}\right)}{2 \Gamma(n+1) \Gamma\left(\frac{1}{2} n+p+1\right)} \\
+\frac{e^{-\left(p+\frac{1}{2} n\right) \pi i} \sqrt{\pi} \Gamma(n+p+1) \Gamma\left(\frac{1}{2} n\right)}{2 \Gamma(n) \Gamma\left(\frac{1}{2} n+p+\frac{1}{2}\right)} x+O\left(x^{2}\right)
\end{gathered}
$$

as $x \rightarrow 0+i 0$. From (4.33) and (4.34), we see that as $x \rightarrow 0+i 0$

$$
\begin{aligned}
P_{n}^{(p, p)}(x)-2 i \frac{e^{\left(p+\frac{1}{2} n\right) \pi i} \cos \left(\frac{1}{2} n \pi\right)}{\pi} \cdot Q_{n}^{(p, p)}(x) & \\
= & -i e^{\frac{1}{2} n \pi i} \frac{\Gamma(n+p+1) \Gamma\left(\frac{1}{2} n\right)}{\sqrt{\pi} \Gamma(n) \Gamma\left(\frac{1}{2} n+p+\frac{1}{2}\right)} x+O\left(x^{2}\right)
\end{aligned}
$$

and consequently our required "recessive" solution at $x=0$ is given by the combination on the left-hand side of (4.35). Therefore, the left-hand side of (4.35) is equivalent to

$$
D_{0}\left(u, \alpha^{*}\right)\left(1-x^{2}\right)^{-\frac{1}{2} p}\left\{\frac{\alpha^{*}-\eta}{\eta\left(\beta-x^{2}\right)}\right\}^{\frac{1}{4}}\left\{\hat{\mathcal{U}}_{\frac{1}{2} u \alpha^{*}, \frac{1}{4}}^{(0)}(2 u \eta)+\hat{\varepsilon}_{0}\left(u, \alpha^{*}, \eta\right)\right\}
$$

for some constant $D_{0}\left(u, \alpha^{*}\right)$. The error bound for $\hat{\varepsilon}_{0}\left(u, \alpha^{*}, \eta\right)$ given by Theorem 2 of [6] holds for all $\eta$ (and $x$ ) under consideration when $n \notin \mathbf{N}$, and for $\eta \in \hat{S}_{\alpha^{*}}^{(1)}$ when $n \in \mathbb{N}$. The constant $D_{0}\left(u, \alpha^{*}\right)$ is found by comparing the behavior of (4.36) at $x=0$ $(\eta=0)$ with (4.35), and invoking (4.26). As a result, we find that

$$
D_{0}\left(u, \alpha^{*}\right)=-i e^{\frac{1}{2} n \pi i}\left(\frac{u-p}{e}\right)^{\frac{1}{2}(u-p)} \frac{\Gamma(n+p+1)}{2^{\frac{1}{2} n} \sqrt{u}(u+p)^{\frac{1}{4}} \Gamma(n+1) \Gamma\left(\frac{1}{2} n+p+\frac{1}{2}\right)} .
$$

Thus, on equating the right-hand side of (4.35) with (4.36) and referring to (4.31), we arrive at our desired approximation 


$$
\begin{aligned}
& P_{n}^{(p, p)}(x)=\left(x^{2}-1\right)^{-\frac{1}{2} p}\left\{\frac{\eta-\alpha^{*}}{\eta\left(x^{2}-\beta\right)}\right\}^{\frac{1}{4}}\left[D _ { 0 } ( u , \alpha ^ { * } ) e ^ { \frac { 1 } { 2 } p \pi i } \left\{\hat{\mathcal{U}}_{\frac{1}{2} u \alpha^{*}, \frac{1}{4}}^{(0)}(2 u \eta)\right.\right. \\
& \left.\left.+\hat{\varepsilon}_{0}\left(u, \alpha^{*}, \eta\right)\right\}+2 i D_{1}\left(u, \alpha^{*}\right) \frac{e^{\left(p+\frac{1}{2} n\right) \pi i} \cos \left(\frac{1}{2} n \pi\right)}{\pi}\left\{\mathcal{U}_{\frac{1}{2} u \alpha^{*}, \frac{1}{4}}^{(1)}(2 u \eta)+\hat{\varepsilon}_{1}\left(u, \alpha^{*}, \eta\right)\right\}\right],
\end{aligned}
$$

which is valid for $\eta \in \hat{S}_{\alpha^{*}}^{(1)}$ when $n \in \mathbf{N}$, and for $\eta \in \hat{S}_{\alpha^{*}}^{(1)} \cup \hat{S}_{\alpha^{*}}^{-}$when $n \notin \mathbf{N}$. An approximation for $P_{n}^{(p, p)}(x)$ which holds for $\eta \in \hat{S}_{\alpha^{*}}^{-}$when $n \in \mathbf{N}$ comes directly from (4.30) and the relation (1.11). Thus an approximation for $P_{n}^{(p, p)}(x)$ is available for all $\eta$ and all $n$ under consideration.

As a final remark both asymptotic solutions appearing in (4.38) are actually valid for $\eta \in \hat{S}_{\alpha^{*}}^{+}$as well as when $n \notin \mathbf{N}$, but (4.38) should not be used for $\eta \in \hat{S}_{\alpha^{*}}^{+}$when $n \notin \mathbf{N}$ since there is severe cancellation in the dominant terms on the right-hand side (since the left-hand side is recessive). However, this is not a problem since (4.28) provides the required approximation for $P_{n}^{(p, p)}(x)$ when $\eta \in \hat{S}_{\alpha^{*}}^{+}$, which is valid for all non-negative $n$.

5. $p$ and $q$ small or large and equal, and $n$ large: the ultraspherical polynomials. As we noted at the beginning of the previous section, when $p=q$ Jacobi's equation takes the form (4.3), and when $u \rightarrow \infty$, has two turning points located at $x= \pm \sqrt{\beta}$ where $\beta$ is defined by (4.4). In this section, we consider the complementary case where these turning points can coalesce (simultaneously) with the double poles at $x= \pm 1$, respectively, but now are bounded away from one another (i.e., bounded away from the origin). Thus the results of this section are valid for $\delta \leq \beta \leq 1$, or equivalently, for $0 \leq \tilde{p} \leq 1-\delta$. Thus we will construct asymptotic expansions which are valid for $n \rightarrow \infty$ and all $p$ (equal to $q$ ) satisfying

$$
0 \leq p \leq A n
$$

where $A$ is an arbitrary positive constant (which is independent of $n$ ).

The analysis for the present case follows similarly to that of $\S 3$. We first observe that the coefficient of $u^{2}$ in equation (4.3) has the behavior

$$
\frac{x^{2}-\beta}{\left(x^{2}-1\right)^{2}}=\frac{\tilde{p}^{2}}{4(x-1)^{2}}+O\left(\frac{1}{x-1}\right)
$$

as $x \rightarrow 1$, and hence the role of $\alpha$ of the general theory given by [4] is played this time by $\tilde{p}$. Thus, if we denote the new independent Liouville variable by $\rho$ and the corresponding dependent variable by $\tilde{W}(\rho)$, then the appropriate transformation is given by

$$
\begin{gathered}
\tilde{W}(\rho)=\left(\frac{d \rho}{d x}\right)^{\frac{1}{2}} w(x), \\
\frac{d \rho}{d x}=\frac{2 \rho}{\left(1-x^{2}\right)}\left\{\frac{x^{2}-\beta}{\tilde{p}^{2}-\rho}\right\}^{\frac{1}{2}},
\end{gathered}
$$

which results in our new equation

$$
\frac{d^{2} \tilde{W}}{d \rho^{2}}=\left\{u^{2}\left(\frac{\tilde{p}^{2}}{4 \rho^{2}}-\frac{1}{4 \rho}\right)+\frac{\tilde{\psi}(\tilde{p}, \rho)}{\rho}-\frac{1}{4 \rho^{2}}\right\} \tilde{W}
$$


where

$$
\tilde{\psi}(\tilde{p}, \rho)=\frac{\rho+4 \tilde{p}^{2}}{16\left(\rho-\tilde{p}^{2}\right)^{2}}+\frac{\left(\tilde{p}^{2}-\rho\right)\left(1-x^{2}\right)\left\{(4 \beta-3) x^{2}+\beta^{2}-2 \beta\right\}}{16 \rho\left(x^{2}-\beta\right)^{3}} .
$$

Integration of (5.4) yields

$$
\int_{\rho}^{\tilde{p}^{2}} \frac{\left(\tilde{p}^{2}-\tau\right)^{\frac{1}{2}}}{2 \tau} d \tau=\int_{\sqrt{\beta}}^{x} \frac{\left(t^{2}-\beta\right)^{\frac{1}{2}}}{1-t^{2}} d t
$$

where the integration limits are chosen so that the turning point $x=\sqrt{\beta}$ of the original equation (4.4) is mapped to the turning point $\rho=\tilde{p}^{2}$ of the new equation (5.5). Note also that the double pole $x=1$ of the original equation (4.4) is mapped to the double pole $\rho=0$ of the new equation (5.5).

Explicit integration of (5.7) gives the following relationship:

$$
\begin{aligned}
& \tilde{p} \ln \left\{\tilde{p}+\left(\tilde{p}^{2}-\rho\right)^{\frac{1}{2}}\right\}-\frac{1}{2} \tilde{p} \ln (\rho)-\left(\tilde{p}^{2}-\rho\right)^{\frac{1}{2}} \\
& \quad=\frac{\tilde{p}}{2} \ln \left\{\frac{(2-\beta) x^{2}-\beta+2 \tilde{p} x\left(x^{2}-\beta\right)^{\frac{1}{2}}}{\beta\left(1-x^{2}\right)}\right\}-\ln \left\{x+\left(x^{2}-\beta\right)^{\frac{1}{2}}\right\}+\frac{1}{2} \ln (\beta) .
\end{aligned}
$$

For simplicity, we shall consider only the right-half plane $|\arg (x)| \leq \frac{1}{2} \pi$, with extension beyond this easily obtained from the continuation formulas (4.9) and (4.10). The branches in (5.7) and (5.8) are taken so that $\rho$ is negative when $x$ lies in the interval $(1, \infty)$ and is a continuous function of $x$ for all other values of $x$ in the half-plane $|\arg (x)| \leq \frac{1}{2} \pi$. With regard to the branch cut associated with $Q_{n}^{(p, p)}(x)$ along the real $x$ axis from 0 to 1 , the corresponding (finite) cut in the $\rho$ plane runs along the positive real axis. With this cut, we specify, for definiteness, that $0 \leq \arg (\rho)<2 \pi$. later:

The following limiting behaviors are deducible from (5.8) and will be required

$$
x=\frac{1}{2} \sqrt{1-\tilde{p}^{2}}\left\{\frac{1+\tilde{p}}{1-\tilde{p}}\right\}^{\frac{1}{2} \tilde{p}} \exp \left\{(-\rho)^{\frac{1}{2}}\right\}\left\{1+O\left(\rho^{-\frac{1}{2}}\right)\right\} \quad(\rho \rightarrow \infty)
$$

and

$$
x=1-\frac{e^{2}}{2\left(1-\tilde{p}^{2}\right)}\left\{\frac{1-\tilde{p}}{1+\tilde{p}}\right\}^{1 / \tilde{p}} \rho+O\left(\rho^{2}\right) \quad(\rho \rightarrow 0) .
$$

The asymptotic expansions furnished by Theorem 3 of [4] now will be written in the form

$$
\begin{aligned}
\tilde{W}_{2 N+1}^{(0)}(u, \tilde{p}, \rho)=2 & \rho^{\frac{1}{2}} J_{p}\left(u \rho^{\frac{1}{2}}\right) \sum_{s=0}^{N} \frac{\tilde{A}_{s}(\tilde{p}, \rho)}{u^{2 s}} \\
& +\frac{2 \rho}{u} J_{p}^{\prime}\left(u \rho^{\frac{1}{2}}\right) \sum_{s=0}^{N-1} \frac{\tilde{B}_{s}(\tilde{p}, \rho)}{u^{2 s}}+\tilde{\varepsilon}_{2 N+1}^{(0)}(u, \tilde{p}, \rho), \\
\tilde{W}_{2 N+1}^{(1)}(u, \tilde{p}, \rho)=\rho^{\frac{1}{2}} & H_{p}^{(1)}\left(u \rho^{\frac{1}{2}}\right) \sum_{s=0}^{N} \frac{\tilde{A}_{s}(\tilde{p}, \rho)}{u^{2 s}} \\
& +\frac{\rho}{u} H_{p}^{(1)^{\prime}}\left(u \rho^{\frac{1}{2}}\right) \sum_{s=0}^{N-1} \frac{\tilde{B}_{s}(\tilde{p}, \rho)}{u^{2 s}}+\tilde{\varepsilon}_{2 N+1}^{(1)}(u, \tilde{p}, \rho)
\end{aligned}
$$


where $\tilde{A}_{0}(\tilde{p}, \rho)=1$, and for $s=1,2,3, \ldots$,

$$
\begin{gathered}
\tilde{B}_{s}(\tilde{p}, \rho)=\left(\rho-\tilde{p}^{2}\right)^{-\frac{1}{2}} \int_{\tilde{p}^{2}}^{\rho}\left(\tau-\tilde{p}^{2}\right)^{-\frac{1}{2}}\left\{\tau \tilde{A}_{s}^{\prime \prime}(\tilde{p}, \tau)+\tilde{A}_{s}^{\prime}(\tilde{p}, \tau)-\tilde{\psi}(\tilde{p}, \tau) \tilde{A}_{s}(\tilde{p}, \tau)\right\} d \tau \\
\tilde{A}_{s}(\tilde{p}, \rho)=-\rho \tilde{B}_{s-1}^{\prime}(\tilde{p}, \rho)+\int_{0}^{\rho} \tilde{\psi}(\tilde{p}, \tau) \tilde{B}_{s-1}(\tilde{p}, \tau) d \tau+\tilde{\lambda}_{s}(\tilde{p}) .
\end{gathered}
$$

As in $\S 3$, we shall select the integration constants $\left\{\tilde{\lambda}_{s}(\tilde{p})\right\}_{s=1}^{N}$ to simplify certain connection coefficients.

The solutions (5.11) and (5.12) should be compared to (3.16) and (3.17), which have the same forms. For the solution (5.11), which is recessive at $x=1(\rho=0)$, we find in a similar manner to the determination of (3.21) and (3.22), that

$$
P_{n}^{(p, p)}(x)=\frac{\tilde{D}_{2 N+1}^{(0)}(u, \tilde{p})}{\left(1-x^{2}\right)^{\frac{1}{2} p} \rho^{\frac{1}{2}}}\left\{\frac{\tilde{p}^{2}-\rho}{x^{2}-\beta}\right\}^{\frac{1}{4}} \tilde{W}_{2 N+1}^{(0)}(u, \tilde{p}, \rho)
$$

where, with the aid of (5.10),

$$
\tilde{D}_{2 N+1}^{(0)}(u, \tilde{p})=\frac{2^{p-1} e^{p} \Gamma(n+p+1)}{\Gamma(n+1)\left(u^{2}-p^{2}\right)^{\frac{1}{2} p}}\left(\frac{u-p}{u+p}\right)^{\frac{1}{2} u}\left[1+\sum_{s=1}^{N} \frac{\tilde{\lambda}_{s}(\tilde{p})}{u^{2 s}}+\tilde{p} \sum_{s=0}^{N-1} \frac{\tilde{B}_{s}(\tilde{p}, 0)}{u^{2 s+1}}\right]^{-1} .
$$

This asymptotic expansion is valid for all $x$ in the half-plane $|\arg (x)| \leq \frac{1}{2} \pi$.

Likewise, for the solution (5.12) which is recessive at $x=\infty(\rho=-\infty)$, we find

$$
Q_{n}^{(p, p)}(x)=\frac{\tilde{D}_{2 N+1}^{(1)}(u, \tilde{p})}{\left(x^{2}-1\right)^{\frac{1}{2} p} \rho^{\frac{1}{2}}}\left\{\frac{\tilde{p}^{2}-\rho}{x^{2}-\beta}\right\}^{\frac{1}{4}} \tilde{W}_{2 N+1}^{(1)}(u, \tilde{p}, \rho)
$$

where, by a matching at $x=1(\rho=0)$, it is found that

$$
\begin{aligned}
\tilde{D}_{2 N+1}^{(1)}(u, \tilde{p})=\pi i \frac{e^{\frac{1}{2} p \pi i} 2^{p-1} \Gamma(n+p+1)}{e^{p} \Gamma(n+2 p+1)}\left(u^{2}-p^{2}\right)^{\frac{1}{2} p}\left(\frac{u+p}{u-p}\right)^{\frac{1}{2} u} \\
\times\left[1+\sum_{s=1}^{N} \frac{\tilde{\lambda}_{s}(\tilde{p})}{u^{2 s}}-\tilde{p} \sum_{s=0}^{N-1} \frac{\tilde{B}_{s}(\tilde{p}, 0)}{u^{2 s+1}}+\tilde{\delta}_{2 N+1}^{(1)}(u, \tilde{p})\right]^{-1}
\end{aligned}
$$

in which

$$
\tilde{\delta}_{2 N+1}^{(1)}(u, \tilde{p})=\lim _{\rho \rightarrow 0}\left\{\rho^{\frac{1}{2}} H_{p}^{(1)}\left(u \rho^{\frac{1}{2}}\right)\right\}^{-1} \tilde{\varepsilon}_{2 N+1}^{(1)}(u, \tilde{p}, \rho) .
$$

Again the expansion (5.17) is valid for all $x$ in the half-plane $|\arg (x)| \leq \frac{1}{2} \pi$.

As an alternative representation for $\tilde{D}_{2 N+1}^{(1)}(u, \tilde{p})$, which does not involve an unknown constant, we compare both sides of (5.17) as $\rho \rightarrow \infty$; with the aid of (1.21) and (5.9), we then find that

$$
\tilde{D}_{2 N+1}^{(1)}(u, \tilde{p})=\pi i e^{\frac{1}{2} p \pi i} \frac{\Gamma\left(u+\frac{1}{2}\right)}{2 \sqrt{u} \Gamma(u)}\left\{\frac{4(u-p)}{u+p}\right\}^{\frac{1}{2} p}\left\{\frac{u^{2}}{u^{2}-p^{2}}\right\}^{\frac{1}{2} u}
$$




$$
\times\left[1+\sum_{s=1}^{N} \frac{\tilde{A}_{s}(\tilde{p}, \infty)}{u^{2 s}}+\sum_{s=0}^{N-1} \frac{\tilde{b}_{s}(\tilde{p}, \infty)}{u^{2 s+1}}\right]^{-1}
$$

where

$$
\tilde{A}_{s}(\tilde{p}, \infty)=\lim _{\rho \rightarrow-\infty} \tilde{A}_{s}(\tilde{p}, \rho), \quad \tilde{b}_{s}(\tilde{p}, \infty)=i \lim _{\rho \rightarrow-\infty} \rho^{\frac{1}{2}} \tilde{B}_{s}(\tilde{p}, \rho)
$$

Next, using (1.28) (with $q=p$ ), (5.15), and (5.17), we find, by following the procedure which yielded (3.29), that

$$
\tilde{D}_{2 N+1}^{(1)}(u, \tilde{p})=\pi i e^{\frac{1}{2} p \pi i}\left\{1+O\left(u^{-2 N-1}\right)\right\} \tilde{D}_{2 N+1}^{(0)}(u, \tilde{p}) .
$$

We then choose the constants $\left\{\tilde{\lambda}_{s}(\tilde{p})\right\}_{s=1}^{N}$ in turn so that

$$
\begin{array}{r}
\left(1+\sum_{s=1}^{N} \frac{\tilde{\lambda}_{s}(\tilde{p})}{u^{2 s}}+\tilde{p} \sum_{s=0}^{N-1} \frac{\tilde{B}_{s}(\tilde{p}, 0)}{u^{2 s+1}}\right)\left(1+\sum_{s=1}^{N} \frac{\tilde{\lambda}_{s}(\tilde{p})}{u^{2 s}}-\tilde{p} \sum_{s=0}^{N-1} \frac{\tilde{B}_{s}(\tilde{p}, 0)}{u^{2 s+1}}\right) \\
=1+O\left(u^{-2 N-1}\right)
\end{array}
$$

(cf. (3.31)). Therefore, from (5.16), (5.18), (5.22), and (5.23), we arrive at our desired simplified forms

$$
\tilde{D}_{2 N+1}^{(0)}(u, \tilde{p})=\frac{2^{p-1} \Gamma(n+p+1)}{[\Gamma(n+2 p+1) \Gamma(n+1)]^{\frac{1}{2}}}\left\{1+O\left(u^{-2 N-1}\right)\right\},
$$

and

$$
\tilde{D}_{2 N+1}^{(1)}(u, \tilde{p})=\pi i e^{\frac{1}{2} p \pi i} \frac{2^{p-1} \Gamma(n+p+1)}{[\Gamma(n+2 p+1) \Gamma(n+1)]^{\frac{1}{2}}}\left\{1+O\left(u^{-2 N-1}\right)\right\} .
$$

Finally, if exact representations for $\tilde{D}_{2 N+1}^{(0)}(u, \tilde{p})$ and $\tilde{D}_{2 N+1}^{(1)}(u, \tilde{p})$ are desired, we can return to (5.16) and (5.20), with the coefficients $\left\{\tilde{\lambda}_{s}(\tilde{p})\right\}_{s=1}^{N},\left\{\tilde{B}_{s}(\tilde{p}, 0)\right\}_{s=0}^{N-1}$, $\left\{\tilde{A}_{s}(\tilde{p}, \infty)\right\}_{s=1}^{N}$, and $\left\{\tilde{b}_{s}(\tilde{p}, \infty)\right\}_{s=0}^{N-1}$ being computed via the formal expansions (cf. (3.36) and (3.44))

$$
\begin{aligned}
&\left(1-\tilde{p}^{2}\right)^{-\frac{1}{2} u \tilde{p}}\left(\frac{1-\tilde{p}}{1+\tilde{p}}\right)^{\frac{1}{2} u}\left(\frac{e}{u}\right)^{u \tilde{p}}\left[\frac{\Gamma\left(u(1+\tilde{p})+\frac{1}{2}\right)}{\Gamma\left(u(1-\tilde{p})+\frac{1}{2}\right)}\right]^{\frac{1}{2}} \\
& \sim 1+\sum_{s=1}^{\infty} \frac{\tilde{\lambda}_{s}(\tilde{p})}{u^{2 s}}+\tilde{p} \sum_{s=0}^{\infty} \frac{\tilde{B}_{s}(\tilde{p}, 0)}{u^{2 s+1}} \\
&\left(1-\tilde{p}^{2}\right)^{-\frac{1}{2} u}\left(\frac{1-\tilde{p}}{1+\tilde{p}}\right)^{\frac{1}{2} u \tilde{p}} \frac{\left[\Gamma\left(u(1+\tilde{p})+\frac{1}{2}\right) \Gamma\left(u(1-\tilde{p})+\frac{1}{2}\right)\right]^{\frac{1}{2}}}{\sqrt{u} \Gamma(u)} \\
& \sim 1+\sum_{s=1}^{\infty} \frac{\tilde{A}_{s}(\tilde{p}, \infty)}{u^{2 s}}+\sum_{s=0}^{\infty} \frac{\tilde{b}_{s}(\tilde{p}, \infty)}{u^{2 s+1}}
\end{aligned}
$$




\section{REFERENCES}

[1] M. Abramowitz and I. A. Stegun, ed., Handbook of Mathematical Functions, Dover, New York, 1965.

[2] R. A. Askey, T. H. Koornwinder And W. Schempp, ed., Special Functions: Group Theoretical Aspects and Applications, Kluwer Academic Pub., Boston, 1984.

[3] P. Baratella and L. Gatteschi, The bounds for the error term of an asymptotic approximation of Jacobi polynomials, in Orthogonal Polynomials and Their Applications, Lecture Notes in Math. 1329, Springer-Verlag Berlin, New York, 1988, pp. 203-221.

[4] W. G. C. Boyd AND T. M. Dunster, Uniform asymptotic solutions of a class of secondorder linear differential equations having a turning point and a regular singularity, with an application to Legendre functions, SIAM J. Math. Anal., 17 (1986), pp. 422-450.

[5] L.-C. Chen And M. E. H. Ismail, On asymptotics of Jacobi polynomials, SIAM J. Math. Anal., 22 (1991), pp. 1442-1449.

[6] T. M. DUNSTER, Uniform asymptotic solutions of second-order linear differential equations having a simple pole and a coalescing turning point in the complex plane, SIAM J. Math. Anal., 25 (1994), pp. 322-353.

[7] D. EllioT, Uniform asymptotic expansions of the Jacobi polynomials and an associated function, Math. Computation, 25 (1971), pp. 309-315.

[8] A. Erdelyi, W. Magnus, F. Oberttinger, and F. Tricomi, Higher Transcendental Functions, 2, McGraw-Hill, New York, 1953.

[9] C. L. Frenzen and R. Wong, A uniform asymptotic expansion of the Jacobi polynomials with error bounds, Canad. J. Math., 37 (1985), pp. 979-1007.

[10] F. W. J. Olver, Asymptotics and Special Functions, Academic Press, New York, 1974; Reprinted by AK Peters, Wellesley, 1997.

[11] — Second-order linear differential equations with two turning points, Phil. Trans. Roy. Soc. London, Ser. A, 278 (1975), pp. 137-174.

[12] J. J. NESTOR, Uniform asymptotic approximations of solutions of second-order linear differential equations, with a coalescing simple turning point and simple pole, Ph.D. thesis, University of Maryland, College Park, MD, 1984.

[13] C. K. QU AND R. Wong, Szegö's conjecture on Lebesgue constants for Legendre series, Pacific J. Math., 135 (1988), pp. 157-188.

[14] G. Szegö, Orthogonal Polynomials, Colloquium Publications, American Mathematical Society, Providence, 1975.

[15] J. Wimp, P. MCCABE, AND J. N. L. Connor, Computation of Jacobi functions of the second kind for use in nearside-farside scattering theory, J. Comp. App. Math., 82 (1997), pp. 447-464.

[16] R. WONG AND J.-M. ZHANG, The asymptotics of a second solution to the Jacobi differential equation, Integral Transforms and Special Functions, 5 (1997), pp. 287-308. 
T. M. DUNSTER 